KEK-TH-1348

\title{
Constraints on Mass Spectrum of Fourth Generation Fermions and Higgs Bosons
}

\author{
Michio Hashimoto* \\ Theory Center, Institute of Particle and Nuclear Studies, \\ High Energy Accelerator Research Organization (KEK), \\ 1-1 Oho, Tsukuba, Ibaraki 305-0801, Japan
}

(Dated: October 28, 2018)

\begin{abstract}
We reanalyze constraints on the mass spectrum of the chiral fourth generation fermions and the Higgs bosons for the standard model (SM4) and the two Higgs doublet model (THDM). We find that the Higgs mass in the SM4 should be larger than roughly the fourth generation up-type quark mass, while the light CP even Higgs mass in the THDM can be smaller. Various mass spectra of the fourth generation fermions and the Higgs bosons are allowed. The phenomenology of the fourth generation models is still rich.
\end{abstract}

PACS numbers: 12.60.Fr, 14.65.Jk, 14.80.Ec, 14.80.Fd

\section{INTRODUCTION}

Repetition of the generation structure of quarks and leptons is a great mystery in particle physics. Although three generation models are widely accepted, the basic principle of the standard model (SM) allows the sequential fourth generation (family) $[1,2]$. Also, the electroweak precision data does not exclude completely existence of the fourth family [3-5]. Since the LHC has a discovery potential for the fourth generation quarks at early stage [6], we may explore this possibility more seriously.

If the fourth generation exists, it is well-known that the condensate of the fourth generation quarks $t^{\prime}$ and $b^{\prime}$ can dynamically trigger the electroweak symmetry breaking (EWSB) [7]. In such a scenario, multiple composite Higgs bosons can naturally emerge as the scalar bound states of $t^{\prime}, b^{\prime}$ and other heavy fermions such as the top quark $t$ and the fourth family leptons $\tau^{\prime}$ and $\nu^{\prime}[8,9]$. When the composite Higgs bosons composed of $t, \tau^{\prime}$ and $\nu^{\prime}$ are too heavy and hence inaccessible at Tevatron and LHC, the effective theory at the $\mathrm{TeV}$ scale will be a two Higgs doublet model (THDM) [10]. Furthermore, if the extra Higgs bosons other than the SM-like Higgs are decoupled [11], the effective theory of the THDM is reduced into the SM with the fourth family (SM4).

In this paper, we study the SM4 and also a THDM with the fourth generation. We assume Dirac-type neutrinos. Models with Majorana mass terms will be studied elsewhere.

The yukawa couplings of the fourth generation have the Landau pole, so that the SM4 or the THDM are applicable up to at most several tens $\mathrm{TeV}$. In this sense, it

*Electronic address: michioh@post.kek.jp is natural to expect the existence of some strong dynamics such as topcolor models [12] behind the SM4/THDM. Nevertheless, we will not impose the compositeness condition $[13,14]$, because we are interested in a wider class of models rather than the Nambu-Jona-Lasino type one.

We reanalyze the stability condition(s) of the Higgs potential for the SM4 and the THDM [15, 16], and also impose the tree level unitarity bounds on the yukawa [17] and Higgs quartic couplings [18-20]. We then find the cutoff $\Lambda$ at which some new physics enters to evade the instability of the Higgs potential or the perturbative description breaks down owing to appearance of some strong dynamics. The cutoff $\Lambda$ should not be so small. Otherwise, the models are not self-contained at the $\mathrm{TeV}$ scale. Besides the theoretical restriction, we take into account the constraints on the oblique parameters [21].

By varying all masses of the fourth generation fermions and the Higgs boson(s) within a reasonable parameter space, we obtain a set of favorable mass spectra. Strong correlations among the masses of the fermions and the Higgs bosons are found. It turns out that the Higgs mass in the SM4 should be larger than roughly the $t^{\prime}$ mass, while the light CP even Higgs mass in the THDM can be smaller because the dynamics of the extra Higgs quartic couplings can stabilize the Higgs potential against the negative contributions of the yukawa couplings. Another noticeable consequence is that the decay channel $\tau^{\prime} \rightarrow \nu^{\prime}+W^{-}$is allowed in a wide parameter space in both of the SM4 and the THDM. The decay channel $t^{\prime} \rightarrow b^{\prime}+W^{(*)}$ is not necessarily excluded. As for the Higgs, a decay channel into a pair of the fourth generation neutrinos is kinematically open in a certain parameter region. Depending on such possibilities, more comprehensive studies should be required.

The paper is organized as follows: In Sec. II, we analyze the SM4. In Sec. III, the THDM is studied. Sec. IV is devoted for summary and discussions. We show the renormalization group equations (RGE's) for the SM4 
and the THDM in Appendix A and B, respectively.

\section{SM4}

Let us study the SM4,

$$
\mathcal{L}_{\mathrm{SM} 4}=\mathcal{L}_{\text {kin }}-\mathcal{L}_{Y}-m_{\phi}^{2}|\phi|^{2}-\lambda|\phi|^{4},
$$

with

$$
\begin{aligned}
\mathcal{L}_{y}= & y_{t} \bar{q}_{L}^{(3)} t_{R} \tilde{\phi}+y_{b} \bar{q}_{L}^{(3)} b_{R} \phi+y_{t^{\prime}} \bar{q}_{L}^{(4)} t_{R}^{\prime} \tilde{\phi}+y_{b^{\prime}} \bar{q}_{L}^{(4)} b_{R}^{\prime} \phi \\
& +y_{\tau} \bar{\ell}_{L}^{(3)} \tau_{R} \phi+y_{\nu^{\prime}} \bar{\ell}_{L}^{(4)} \nu_{R}^{\prime} \tilde{\phi}+y_{\tau^{\prime}} \bar{\ell}_{L}^{(4)} \tau_{R}^{\prime} \phi,
\end{aligned}
$$

where $\phi$ represents the Higgs doublet field, $\tilde{\phi}$ is defined by $\tilde{\phi} \equiv i \tau_{2} \phi^{*}$, and $q^{(i)}$ and $\ell^{(i)}$ denote the $i$-th family doublet of quarks and leptons, respectively. We take into account the yukawa couplings of the third and fourth generations, and ignore other yukawa couplings as well as the neutrino masses other than $\nu^{\prime}$. As explicitly shown in $\mathcal{L}_{y}$, we simply assumed the Dirac-type neutrinos.

The RGE's for the yukawa and Higgs quartic couplings are well-known $[22,23]$. We show a set of the RGE's for the gauge, yukawa and Higgs-quartic couplings at the one-loop approximation in Appendix A.

We explore the cutoff scale $\Lambda$ of the SM4 at which some new physics or nonperturbative dynamics emerges. The point is that the yukawa coupling has the Landau pole at a certain energy scale $\Lambda_{y}$ and only an intermediate mass range of the Higgs boson is allowed by the triviality and instability bounds. Before the full one-loop calculation, we schematically describe nature of the RGE's.

Let us solve analytically the RGE's under the following crude approximation.

The electroweak gauge couplings are negligible. Although the QCD coupling is not so small, it behaves like a constant in the energy scale $\mathcal{O}(1-10) \mathrm{TeV}$. On the other hand, the yukawa couplings for the fourth generation run very quickly and diverge at the Landau pole. Thus we may ignore all of the gauge couplings at the zeroth approximation. For simplicity, we may neglect $y_{t}$ and also assume that all of the fourth generation yukawa couplings are the same as $y_{4}$, although it is unrealistic because owing to a relatively heavy Higgs, the $T$-parameter constraint requires appropriate mass differences of the fourth generation fermions, which will be taken into account in the full analysis of the one-loop RGE's.

Under the above crude approximation, the RGE for $y_{4}$ is given by

$$
\left(16 \pi^{2}\right) \mu \frac{\partial}{\partial \mu} y_{4}=8 y_{4}^{3},
$$

and the solution is immediately found as

$$
\frac{1}{y_{4}^{2}(\mu)}-\frac{1}{y_{4}^{2}\left(\mu_{0}\right)}=-\frac{1}{\pi^{2}} \ln \mu / \mu_{0},
$$

where $\mu_{0}$ is an arbitrary scale. The universal fermion mass $m_{4}$ is defined by $m_{4}=y_{4}\left(\mu=m_{4}\right) v / \sqrt{2}$. By definition of the Landau pole $\Lambda_{y}, 1 / y_{4}^{2}\left(\mu=\Lambda_{y}\right)=0$ and then we obtain the relation between $\Lambda_{y}$ and $m_{4}$ as

$$
\Lambda_{y}=m_{4} e^{\frac{v^{2} \pi^{2}}{2 m_{4}^{2}}}
$$

where $v(=246 \mathrm{GeV})$ is the vacuum expectation value (VEV) of the Higgs. Numerically, it yields

$$
\Lambda_{y}=8(10) \mathrm{TeV}, 3(3) \mathrm{TeV}, 2(2) \mathrm{TeV},
$$

for

$$
m_{4}=300 \mathrm{GeV}, 400 \mathrm{GeV}, 500 \mathrm{GeV} .
$$

Compared with the full one-loop calculation (the values in the parentheses), the approximation works in fact.

As for the Higgs sector, within the above crude approximation, the RGE for $\lambda$ is

$$
\left(16 \pi^{2}\right) \mu \frac{\partial}{\partial \mu} \lambda=24 \lambda^{2}+\left(32 y_{4}^{2} \lambda-16 y_{4}^{4}\right) \theta\left(\mu-m_{4}\right),
$$

where we explicitly treated the fermion contributions to the $\beta$-function in the $\theta$-function, i.e., below the threshold of $m_{4}$, the theory is matched to the SM without the fourth generation. We can easily find that the following quantity is the RGE invariant:

$$
\eta \equiv \frac{\tilde{\lambda}(\mu)-\xi_{+}}{\tilde{\lambda}(\mu)-\xi_{-}}\left(y_{4}(\mu)\right)^{-2 \sqrt{7}}, \quad\left(\mu>\max \left(m_{4}, m_{\phi^{0}}\right)\right),
$$

where $m_{\phi^{0}}\left(=\sqrt{2 \lambda\left(\mu=m_{\phi^{0}}\right)} v\right)$ is the mass of the physical Higgs boson $\phi^{0}$ and also

$$
\tilde{\lambda}(\mu) \equiv \frac{\lambda(\mu)}{y_{4}^{2}(\mu)}, \quad \xi_{ \pm} \equiv \frac{-1 \pm \sqrt{7}}{3} .
$$

Note that when $\eta>0, \lambda$ goes to infinity at the scale $\Lambda_{\lambda}\left[=\Lambda_{y} \exp \left(-\pi^{2} \eta^{1 / \sqrt{7}}\right)\right]$, while it does to zero at the scale $\Lambda_{\text {inst }}\left[=\Lambda_{y} \exp \left(-\pi^{2}\left\{\xi_{-} \eta / \xi_{+}\right\}^{1 / \sqrt{7}}\right)\right]$, when $\eta<0$.

For $m_{\phi^{0}}<m_{4}$, the RGE for $\lambda$ develops only by the $\lambda^{2}$-term in the region $m_{\phi^{0}}<\mu<m_{4}$, so that it does not encounter instability in this region. For $m_{\phi^{0}}>m_{4}$, we do not need to care about the above threshold effects. Then, in terms of $m_{4}$ and $m_{\phi^{0}}$, the scale $\Lambda_{\text {inst }}$ at which $\lambda\left(\mu=\Lambda_{\text {inst }}\right)=0$ is given by

$$
\Lambda_{\text {inst }}=m_{4} \exp \left[\frac{\pi^{2} v^{2}}{2 m_{4}^{2}}\left\{1-\left(\frac{1-\zeta_{1} \frac{m_{\phi^{0}}^{2}}{8 m_{4}^{2}}}{1+\zeta_{2} \frac{m_{\phi^{0}}^{2}}{8 m_{4}^{2}}}\right)^{\frac{1}{\sqrt{7}}}\right\}\right],
$$



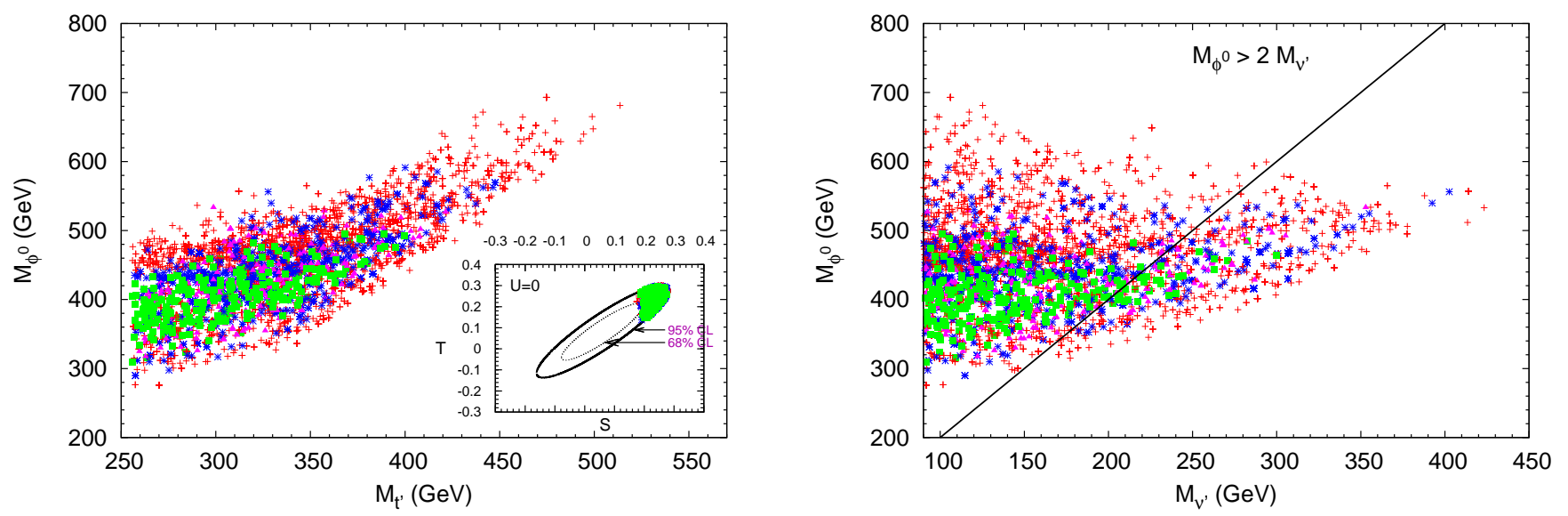

FIG. 1: Scatter plots of $M_{t^{\prime}}$ v.s. $M_{\phi^{0}}$ (left) and $M_{\nu^{\prime}}$ v.s. $M_{\phi^{0}}$ (right). The data points are the same in both figures. We varied $256 \mathrm{GeV}<M_{t^{\prime}}<552 \mathrm{GeV}, 255 \mathrm{GeV}<M_{b^{\prime}}<552 \mathrm{GeV}, 100.8 \mathrm{GeV}<M_{\tau^{\prime}}<1.23 \mathrm{TeV}, 90.3 \mathrm{GeV}<M_{\nu^{\prime}}<1.23 \mathrm{TeV}$, and $114 \mathrm{GeV}<M_{\phi^{0}}<873 \mathrm{GeV}$, without any prejudice. We took into account all of the 40 patterns of the mass spectrum of the fermions and the corresponding threshold effects. The red, blue, magenta and green points correspond to the cutoff $\Lambda$, $2 \mathrm{TeV} \leq \Lambda<3 \mathrm{TeV}, 3 \mathrm{TeV} \leq \Lambda<4 \mathrm{TeV}, 4 \mathrm{TeV} \leq \Lambda<5 \mathrm{TeV}$ and $\Lambda \geq 5 \mathrm{TeV}$, respectively. Below the cutoff scale $\Lambda$, the Higgs potential is stable and the perturbation is applicable. The data points are within the $95 \%$ C.L. limit of the $S$ and $T$ parameters. In the inset of the left figure, we showed the $(S, T)$-contour and the data points which we used for the scatter plot.

with

$$
\begin{aligned}
& \zeta_{1} \equiv \frac{\sqrt{7}+1}{1+\frac{3 m_{\phi^{0}}^{2}}{4 \pi^{2} v^{2}} \ln \frac{m_{\phi^{0}}}{m_{4}}}, \\
& \zeta_{2} \equiv \frac{\sqrt{7}-1}{1+\frac{3 m_{\phi^{0}}^{2}}{4 \pi^{2} v^{2}} \ln \frac{m_{\phi^{0}}}{m_{4}}},
\end{aligned}
$$

for $m_{\phi^{0}}<m_{4}$, and

$$
\Lambda_{\text {inst }}=m_{4} \exp \left[\frac{\pi^{2} v^{2}}{2 m_{4}^{2}}-t_{\phi^{0}}\left(\frac{1-\frac{(\sqrt{7}+1) m_{\phi^{0}}^{2}}{4 \pi^{2} v^{2}} t_{\phi^{0}}}{1+\frac{(\sqrt{7}-1) m_{\phi^{0}}^{2}}{4 \pi^{2} v^{2}} t_{\phi^{0}}}\right)^{\frac{1}{\sqrt{7}}}\right],
$$

with

$$
t_{\phi^{0}} \equiv \ln \frac{\Lambda_{y}}{m_{\phi^{0}}}=\ln \frac{m_{4}}{m_{\phi^{0}}}+\frac{v^{2} \pi^{2}}{2 m_{4}^{2}},
$$

for $m_{\phi^{0}}>m_{4}$. Similarly, the Landau pole $\Lambda_{\lambda}$ for $\lambda$, i.e., $\lambda\left(\mu=\Lambda_{\lambda}\right)=\infty$, is given by

$$
\Lambda_{\lambda}=m_{\phi^{0}} \exp \left[t_{\phi^{0}}\left\{1-\left(1-\zeta_{\lambda}\right)^{\frac{1}{\sqrt{7}}}\right\}\right]
$$

with

$$
\zeta_{\lambda} \equiv \frac{2 \sqrt{7}}{\frac{3 m_{\phi^{0}}^{2} t_{\phi^{0}}}{2 \pi^{2} v^{2}}+\sqrt{7}+1}
$$

for $m_{\phi^{0}}>m_{4}$. We find that the solution $\Lambda_{\lambda}$ for $m_{\phi^{0}}<$ $m_{4}$ is phenomenologically unacceptable.

Numerically, with fixing $m_{4}=300 \mathrm{GeV}$, we find

$$
\Lambda_{\text {inst }}=0.44(0.44) \mathrm{TeV}, 0.71(0.74) \mathrm{TeV}, 1.6(2.1) \mathrm{TeV} \text {, }
$$

for

$$
m_{\phi^{0}}=200 \mathrm{GeV}, 300 \mathrm{GeV}, 400 \mathrm{GeV},
$$

and

$$
\Lambda_{\lambda}=4.3(3.7) \mathrm{TeV}, 2.5(2.4) \mathrm{TeV}, 2.1(2.1) \mathrm{TeV},
$$

for

$$
m_{\phi^{0}}=500 \mathrm{GeV}, 600 \mathrm{GeV}, 700 \mathrm{GeV},
$$

where the values in the parentheses are the full one-loop results. The approximation works well.

In passing, the compositeness conditions [13], $1 / y_{4}^{2} \rightarrow$ 0 and $\lambda / y_{4}^{4} \rightarrow 0$, require $\eta=0$ and then we get the relation between $m_{4}$ and $m_{\phi^{0}}$;

$$
\frac{m_{\phi^{0}}^{2}}{2 \pi^{2} v^{2}} t_{\phi^{0}}=\xi_{+}, \quad\left(m_{\phi^{0}}>m_{4}\right) .
$$

Numerically, it gives

$$
m_{\phi^{0}}=480 \mathrm{GeV}, 583 \mathrm{GeV}, 713 \mathrm{GeV},
$$



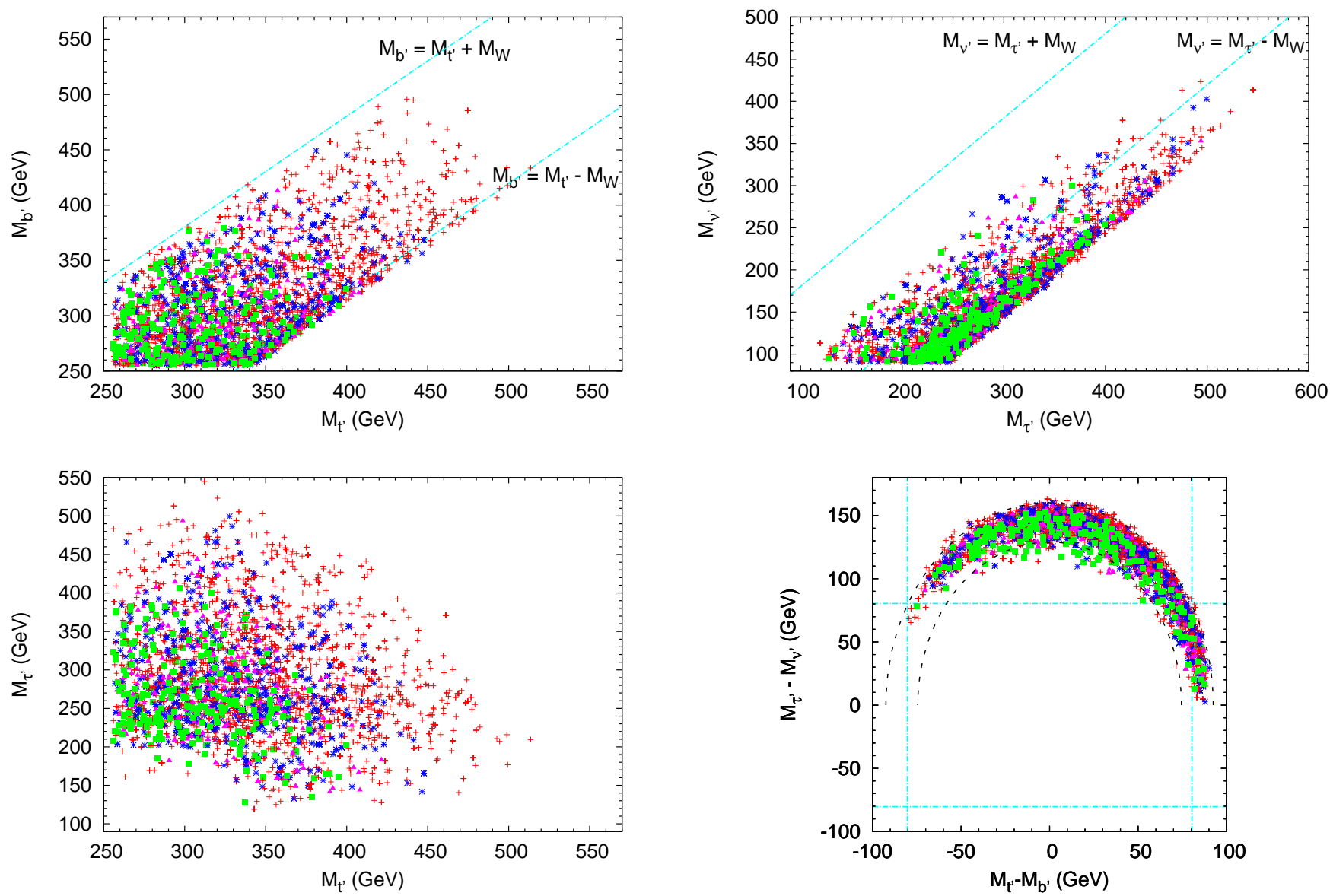

FIG. 2: $M_{t^{\prime}}$ v.s. $M_{b^{\prime}}$ (upper left), $M_{\tau^{\prime}}$ v.s. $M_{\nu^{\prime}}$ (upper right). $M_{t^{\prime}}$ v.s. $M_{\tau^{\prime}}$ (lower left) and $M_{t^{\prime}}-M_{b^{\prime}}$ v.s. $M_{\tau^{\prime}}-M_{\nu^{\prime}}$ (lower right). The data points are the same as those in Fig. 1. In the upper figures, the blue dashed lines correspond to $M_{b^{\prime}\left(\nu^{\prime}\right)}=M_{t^{\prime}\left(\tau^{\prime}\right)} \pm M_{W}$. The blue lines in the lower right correspond to $\pm M_{W}$. Notice that the decay channels $t^{\prime} \rightarrow b^{\prime}+W^{+}$ and $\tau^{\prime} \rightarrow \nu^{\prime}+W^{-}$are kinematically allowed in the parameter regions $M_{t^{\prime}}-M_{b^{\prime}}>M_{W}$ and $M_{\tau^{\prime}}-M_{\nu^{\prime}}>M_{W}$, respectively. In the lower right, the dashed semicircles correspond to the relation (26) with $M_{\phi^{0}}=300,500 \mathrm{GeV}$.

for

$$
m_{4}=300 \mathrm{GeV}, 350 \mathrm{GeV}, 400 \mathrm{GeV} \text {. }
$$

We now proceed to perform the full analysis of the one-loop RGE's.

For a quark $q$, we read the $\overline{\mathrm{MS}}$-mass via [24]

$$
\hat{m}_{q}\left(\mu=\hat{m}_{q}\right)=M_{q}\left(1-\frac{4 \alpha_{s}}{3 \pi}+\mathcal{O}\left(\alpha_{s}^{2}\right)\right),
$$

where $M_{q}$ and $\hat{m}_{q}$ denote the pole and $\overline{\mathrm{MS}}$-masses, respectively. We used $\alpha_{s}\left(M_{Z}\right)=0.118$. For leptons and the Higgs, the tree level formula is utilized. We vary the fermion masses [24], $256 \mathrm{GeV}<M_{t^{\prime}}<\sqrt{8 \pi / 5} v$, $255 \mathrm{GeV}<M_{b^{\prime}}<\sqrt{8 \pi / 5} v, 100.8 \mathrm{GeV}<M_{\tau^{\prime}}<\sqrt{8 \pi} v$, and $90.3 \mathrm{GeV}<M_{\nu^{\prime}}<\sqrt{8 \pi} v$ without any prejudice, where $\sqrt{8 \pi / 5} v \simeq 552 \mathrm{GeV}$ and $\sqrt{8 \pi} v \simeq 1.23 \mathrm{TeV}$ are the perturbative unitarity bounds for quarks and leptons, respectively [17]. We took into account all of the 40 patterns of the mass spectrum of the fermions ${ }^{1}$ and the corresponding threshold effects. For the Higgs mass, we survey the parameter space, $114 \mathrm{GeV}<M_{\phi^{0}}<\sqrt{4 \pi} v(\simeq$ $873 \mathrm{GeV})$. Imposing the perturbative unitarity bounds on all yukawa couplings, and the stability and triviality bound on $\lambda, 0<\lambda(\mu)<2 \pi$, we can estimate the theo-

\footnotetext{
1 Owing to the mass bounds $M_{t^{\prime}}, M_{b^{\prime}}>M_{t}$, the possible patterns are reduced into 40 from $5 !=120$.
} 
retical cutoff scale $\Lambda$ for the SM4.

We also take into account the constraints from the oblique parameters [21]. In order to suppress the $S$ parameter, $M_{t^{\prime}}>M_{b^{\prime}}$ and/or $M_{\tau^{\prime}}>M_{\nu^{\prime}}$ are favorable. Although it increases the $T$-parameter, this is rather nice, because a relatively heavy Higgs pulls down $T[3,5]$. As for estimate of $S$ and $T$, we follow the LEP EWWG [25]. We obtain the central value as $(S, T)=(0.06,0.08)$, where the SM point is normalized to $(S, T)=(0,0)$ and the top mass $M_{t}=173.1 \mathrm{GeV}$ and the reference Higgs mass $M_{\phi^{0}, \text { ref }}=117 \mathrm{GeV}$ are used. The relevant experimental values in the estimate are $M_{Z}=91.1875 \pm 0.0021 \mathrm{GeV}, \Delta \alpha_{\text {had }}^{(5)}=0.02758 \pm 0.00035$, $M_{W}=80.399 \pm 0.0025 \mathrm{GeV}, \sin ^{2} \theta_{\text {eff }}=0.23153 \pm 0.00016$, and $\Gamma_{\ell}=83.985 \pm 0.086 \mathrm{MeV}[26]$.

In Fig. 1, we depict scatter plots $M_{t^{\prime}}$ v.s. $M_{\phi^{0}}$ and $M_{\nu^{\prime}}$ v.s. $M_{\phi^{0}}$ within the $95 \%$ C.L. limit of the $(S, T)$ constraint. In each point, the fermion masses are different. We also showed the $(S, T)$-contour and the data points in the inset of the left of Fig. 1. For consistency of the model, the cutoff scale should not be so small. In the figure, we took the cutoff $\Lambda \geq 2 \mathrm{TeV}$.

We find that the theoretical lower bound of the Higgs mass is $M_{\phi^{0}} \gtrsim M_{t^{\prime}}$, when $\Lambda \geq 2 \mathrm{TeV}$. (If we take $\Lambda \geq$ $1 \mathrm{TeV}$, slightly lower values of $M_{\phi^{0}}$ are allowed, $M_{\phi^{0}} \gtrsim$ $M_{t^{\prime}}-50 \mathrm{GeV}$.) Note that the Higgs production via the gluon fusion process is considerably enhanced owing to the loop effects of $t^{\prime}$ and $b^{\prime}$. For example, the cross section $\sigma_{g g \rightarrow \phi^{0}}$ for $M_{t^{\prime}}=M_{b^{\prime}}=0.4 \mathrm{TeV}$ and $M_{\phi^{0}}=0.5 \mathrm{TeV}$ increases by a factor of 5 . Depending on the masses of $t^{\prime}, b^{\prime}$ and $\phi^{0}$, the enhancement factor varies from 5 to 9. Consequently, a wider mass range of $M_{\phi^{0}}$ should be excluded at Tevatron. This potentially excluded mass range is fairly lower than the above Higgs mass bound, however [27].

In addition, the right of Fig. 1 clearly shows that the decay channel $\phi^{0} \rightarrow \bar{\nu}^{\prime} \nu^{\prime}$ is opened in a favorable parameter space. The importance of this process has been emphasized in Ref. [5], i.e., the new signal via $\phi^{0} \rightarrow \bar{\nu}^{\prime} \nu^{\prime} \rightarrow 4 \ell+\not \ell$, where $\not \subset$ is the missing energy, can be comparable to the rate for $\phi^{0} \rightarrow Z Z \rightarrow 4 \ell$. Moreover, we find that there is a parameter region where $\phi^{0} \rightarrow \bar{\tau}^{\prime} \tau^{\prime}$ is also kinematically allowed. In fact, several scenarios are possible. We show data samples in Table I.

The constraints from the oblique parameters cause strong correlations between $M_{t^{\prime}}$ and $M_{b^{\prime}}$ and also between $M_{\tau^{\prime}}$ and $M_{\nu^{\prime}}$, as shown in the upper left and right of Fig. 2. The lower left of Fig. 2 suggests that there is no correlation between $M_{t^{\prime}}$ and $M_{\tau^{\prime}}$, as expected. On the other hand, the fermion mass differences are strongly correlated, as shown in the lower right of Fig. 2. This essen- tially corresponds to the constraint of the $T$-parameter,

$$
\begin{aligned}
& \frac{3\left(M_{t^{\prime}}-M_{b^{\prime}}\right)^{2}}{M_{W}^{2}}+\frac{\left(M_{\tau^{\prime}}-M_{\nu^{\prime}}\right)^{2}}{M_{W}^{2}} \\
& \approx(1.3-2.0)+1.4 \ln \frac{M_{\phi^{0}}}{M_{\phi^{0}, \text { ref }}} .
\end{aligned}
$$

We depicted it with $M_{\phi^{0}}=300 \mathrm{GeV}, 500 \mathrm{GeV}$ and $M_{\phi^{0}, \text { ref }}=117 \mathrm{GeV}$ in the semicircles of the lower right of Fig. 2. The $S$-parameter constraint also suggests that the parameter region $M_{\tau^{\prime}}>M_{\nu^{\prime}}$ and $M_{t^{\prime}}>M_{b^{\prime}}$ is favorable. (Within the $95 \%$ C.L. limit, $M_{t^{\prime}}<M_{b^{\prime}}$ is also possible with paying cost of a worse $\chi^{2}$, as shown in the lower right of Fig. 2. )

We emphasize that in a wide parameter region, we find $M_{\tau^{\prime}}>M_{\nu^{\prime}}+M_{W}$, i.e., the decay channel $\tau^{\prime} \rightarrow \nu^{\prime}+W^{-}$ is allowed. (The situation is unchanged, even if we take $\Lambda \geq 1 \mathrm{TeV}$.) Also, $t^{\prime} \rightarrow b^{\prime}+W^{(*)}$ is possible. These do not necessarily contradict the results in Ref. [5]: Since the Higgs is inevitably heavy in our approach, the $T$ parameter constraint requires a larger mass difference of the fermions than that of Ref. [5]. (The $\chi^{2}$ is a bit worse, however.)

The implications of Figs. 1-2 are obvious: If $t^{\prime}$ and/or $b^{\prime}$ are discovered at the Tevatron and/or LHC, the Higgs mass will be suggested under the assumption of the SM4. On the other hand, if the LHC excludes $M_{t^{\prime}, b^{\prime}} \lesssim 500 \mathrm{GeV}$, only few points survive when we take $\Lambda \geq 2 \mathrm{TeV}$. I.e., a model with the cutoff $\Lambda<2 \mathrm{TeV}$ or a nonperturbative regime will be left to be explored.

\section{TWO HIGGS DOUBLET MODEL}

\section{A. Model}

Let us consider the THDM with the fourth generation:

$$
\mathcal{L}_{\mathrm{THDM}}=\mathcal{L}_{\text {kin }}-\mathcal{L}_{Y}-V,
$$

where $\mathcal{L}_{\text {kin }}$ represents the kinetic terms of the fermions, the Higgs fields and the SM gauge fields, $\mathcal{L}_{Y}$ denotes the yukawa couplings between fermions and the Higgs fields, and $V$ is the Higgs potential. The yukawa sector, in particular the neutrino one, is model-dependent. For simplicity, we assume that the neutrinos have the Dirac masses. A model with Majorana neutrinos should be considered separately. This is, however, out of scope in this paper.

We define the THDM of the type II (THDM II) with the Dirac neutrinos as follows: One Higgs doublet $\left(\Phi_{1}\right)$ couples to the down-type quarks and charged leptons, while the other $\left(\Phi_{2}\right)$ does to the up-type quarks and neu- 


\begin{tabular}{c||c|cc|cc|c|cc|}
\hline & $M_{\phi^{0}}$ & $M_{t^{\prime}}$ & $M_{b^{\prime}}$ & $M_{\nu^{\prime}}$ & $M_{\tau^{\prime}}$ & $\Lambda$ & $S$ & $T$ \\
\hline \hline (a1) & 0.47 & 0.36 & 0.34 & 0.092 & 0.23 & 3.5 & 0.20 & 0.22 \\
(a2) & 0.59 & 0.45 & 0.42 & 0.092 & 0.24 & 2.3 & 0.21 & 0.22 \\
\hline (b1) & 0.45 & 0.32 & 0.36 & 0.098 & 0.23 & 8.0 & 0.23 & 0.27 \\
(b2) & 0.55 & 0.40 & 0.41 & 0.13 & 0.28 & 3.4 & 0.24 & 0.22 \\
\hline (c1) & 0.51 & 0.44 & 0.36 & 0.10 & 0.17 & 2.1 & 0.24 & 0.28 \\
(c2) & 0.65 & 0.50 & 0.42 & 0.097 & 0.18 & 2.1 & 0.25 & 0.29 \\
\hline (d1) & 0.39 & 0.32 & 0.34 & 0.093 & 0.23 & 2.4 & 0.21 & 0.23 \\
(d2) & 0.55 & 0.43 & 0.41 & 0.13 & 0.28 & 2.1 & 0.24 & 0.23 \\
\hline (e1) & 0.49 & 0.36 & 0.34 & 0.26 & 0.40 & 2.1 & 0.26 & 0.24 \\
\hline
\end{tabular}

TABLE I: Data samples for several scenarios. The mass unit is TeV. For (a1), (b1), (c1), (d1) and (e1), we took the mass bounds $M_{t^{\prime}}>311 \mathrm{GeV}$ and $M_{b^{\prime}}>338 \mathrm{GeV}$ [28], whereas we did $M_{t^{\prime}, b^{\prime}}>400 \mathrm{GeV}$ for (a2), (b2), (c2) and (d2). For all samples, we took $\Lambda \geq 2 \mathrm{TeV}$. The criterion for (a1) and (a2) is the $\chi^{2}$-minimum. Similarly, (b1) and (b2) have the largest $\Lambda$ within the $95 \%$ C.L. limit of the $(S, T)$-constraints. For (c1) and (c2), $t^{\prime} \rightarrow b^{\prime}+W^{+}$is possible. The samples (d1) and (d2) are most favorable data for $M_{\phi^{0}}<2 M_{\tau^{\prime}}$, while (e1) is for $M_{\phi^{0}}<2 M_{\nu^{\prime}}$. We do not have the data sample with $M_{t^{\prime}, b^{\prime}}>400 \mathrm{GeV}$ and $M_{\phi^{0}}<2 M_{\nu^{\prime}}$.

tral leptons, i.e.,

$$
\begin{aligned}
\mathcal{L}_{Y} & =\sum_{i, j=1}^{4} Y_{U}^{i j} \bar{q}_{L}^{(i)} u_{R}^{(j)} \tilde{\Phi}_{2}+\sum_{i, j=1}^{4} Y_{D}^{i j} \bar{q}_{L}^{(i)} d_{R}^{(j)} \Phi_{1} \\
& +\sum_{i, j=1}^{4} Y_{N}^{i j} \bar{\ell}_{L}^{(i)} \nu_{R}^{(j)} \tilde{\Phi}_{2}+\sum_{i, j=1}^{4} Y_{E}^{i j} \bar{\ell}_{L}^{(i)} e_{R}^{(j)} \Phi_{1}
\end{aligned}
$$

where $u_{R}^{(j)}$ represents the right-handed up-type quark of the $j$-th family and the definitions of $d_{R}^{(j)}$ etc. are then self-evident.

The Higgs potential is

$$
\begin{aligned}
V & \left.=m_{1}^{2} \Phi_{1}^{\dagger} \Phi_{1}+m_{2}^{2} \Phi_{2}^{\dagger} \Phi_{2}+m_{12}^{2}\left(\Phi_{1}^{\dagger} \Phi_{2}+\text { (h.c. }\right)\right) \\
& +\lambda_{1}\left(\Phi_{1}^{\dagger} \Phi_{1}\right)^{2}+\lambda_{2}\left(\Phi_{2}^{\dagger} \Phi_{2}\right)^{2}+\lambda_{3}\left(\Phi_{1}^{\dagger} \Phi_{1}\right)\left(\Phi_{2}^{\dagger} \Phi_{2}\right) \\
& +\lambda_{4}\left(\Phi_{1}^{\dagger} \Phi_{2}\right)\left(\Phi_{2}^{\dagger} \Phi_{1}\right)+\frac{1}{2} \lambda_{5}\left(\left(\Phi_{1}^{\dagger} \Phi_{2}\right)\left(\Phi_{1}^{\dagger} \Phi_{2}\right)+(\text { h.c. })\right),
\end{aligned}
$$

where we do not consider the hard $Z_{2}$-breaking terms. Owing to the (softly broken) $Z_{2}$-symmetry, the tree-level FCNC is absent [29].

We do not consider $\mathrm{CP}$ violation in the (tree-level) Higgs sector and hence will take all parameters in the Higgs potential $V$ to be real, so that there are eight parameters. In the yukawa sector, we assume that the mixing terms between the fourth generation and the others are absent, i.e., $Y_{U}^{4 k}=Y_{U}^{k 4}=y_{t^{\prime}} \delta_{4 k}, Y_{D}^{4 k}=Y_{D}^{k 4}=y_{b^{\prime}} \delta_{4 k}$,
$Y_{N}^{4 k}=Y_{N}^{k 4}=y_{\nu^{\prime}} \delta_{4 k}$, and $Y_{E}^{4 k}=Y_{E}^{k 4}=y_{\tau^{\prime}} \delta_{4 k}$. We can then reduce number of parameters.

When the EWSB occurs, three $\left(G^{0}\right.$ and $\left.G^{ \pm}\right)$of the eight scalar degrees of freedom are eaten by the weak gauge bosons. The physical mass spectrum then contains two CP even Higgs bosons $h$ and $H$ defined by $M_{h}<M_{H}$, one CP odd Higgs $A$, and the charged Higgs pair $H^{ \pm}$, so that the original Higgs fields are written in terms of the physical degrees of freedom as follows;

$$
\Phi_{1}=\frac{1}{\sqrt{2}}\left(\begin{array}{c}
\sqrt{2}\left(c_{\beta} G^{+}-s_{\beta} H^{+}\right) \\
c_{\beta} v-s_{\alpha} h+c_{\alpha} H+i\left(c_{\beta} G^{0}-s_{\beta} A\right)
\end{array}\right),
$$

and

$$
\Phi_{2}=\frac{1}{\sqrt{2}}\left(\begin{array}{c}
\sqrt{2}\left(s_{\beta} G^{+}+c_{\beta} H^{+}\right) \\
s_{\beta} v+c_{\alpha} h+s_{\alpha} H+i\left(s_{\beta} G^{0}+c_{\beta} A\right)
\end{array}\right),
$$

where $\alpha$ is the mixing angle between $h$ and $H$ and the ratio of the VEVs of the two Higgs fields is defined by $\tan \beta$. We also used the notations $s_{\beta} \equiv \sin \beta, c_{\beta} \equiv \cos \beta$, and etc..

It is convenient to express the quartic couplings $\lambda_{1-5}$ through the Higgs masses, the soft $Z_{2}$-breaking term $m_{12}^{2}$, and the mixing angles $\alpha$ and $\beta$ :

$$
\begin{aligned}
2 \lambda_{1} v^{2} & =\frac{c_{\alpha}^{2} M_{H}^{2}+s_{\alpha}^{2} M_{h}^{2}}{c_{\beta}^{2}}-M^{2} \tan ^{2} \beta \\
2 \lambda_{2} v^{2} & =\frac{s_{\alpha}^{2} M_{H}^{2}+c_{\alpha}^{2} M_{h}^{2}}{s_{\beta}^{2}}-M^{2} \tan ^{-2} \beta \\
\lambda_{3} v^{2} & =\frac{s_{2 \alpha}}{s_{2 \beta}}\left(M_{H}^{2}-M_{h}^{2}\right)+2 M_{H^{ \pm}}^{2}-M^{2} \\
\lambda_{4} v^{2} & =M_{A}^{2}-2 M_{H^{ \pm}}^{2}+M^{2} \\
\lambda_{5} v^{2} & =-M_{A}^{2}+M^{2}
\end{aligned}
$$

where we used $M^{2}$,

$$
M^{2} \equiv \frac{-m_{12}^{2}}{s_{\beta} c_{\beta}}
$$

instead of $m_{12}^{2}$. Since we consider a general THDM, all quartic couplings are independent and hence free from the MSSM relations [10].

The decoupling limit of the extra Higgs corresponds to $M_{h}^{2} \sim \mathcal{O}\left(v^{2}\right)$ and $M_{H, A, H^{ \pm}}^{2} \sim M^{2} \gg v^{2}$ [11], where $M^{2}$ is independent of the quartic couplings $\lambda_{1-5}$ and thus can be taken as some high scale without contradict against the perturbative unitarity bound. In this case, $\sin (\beta-$ $\alpha) \simeq 1$ is also derived. The low energy effective theory in this limit is reduced into the SM4. 

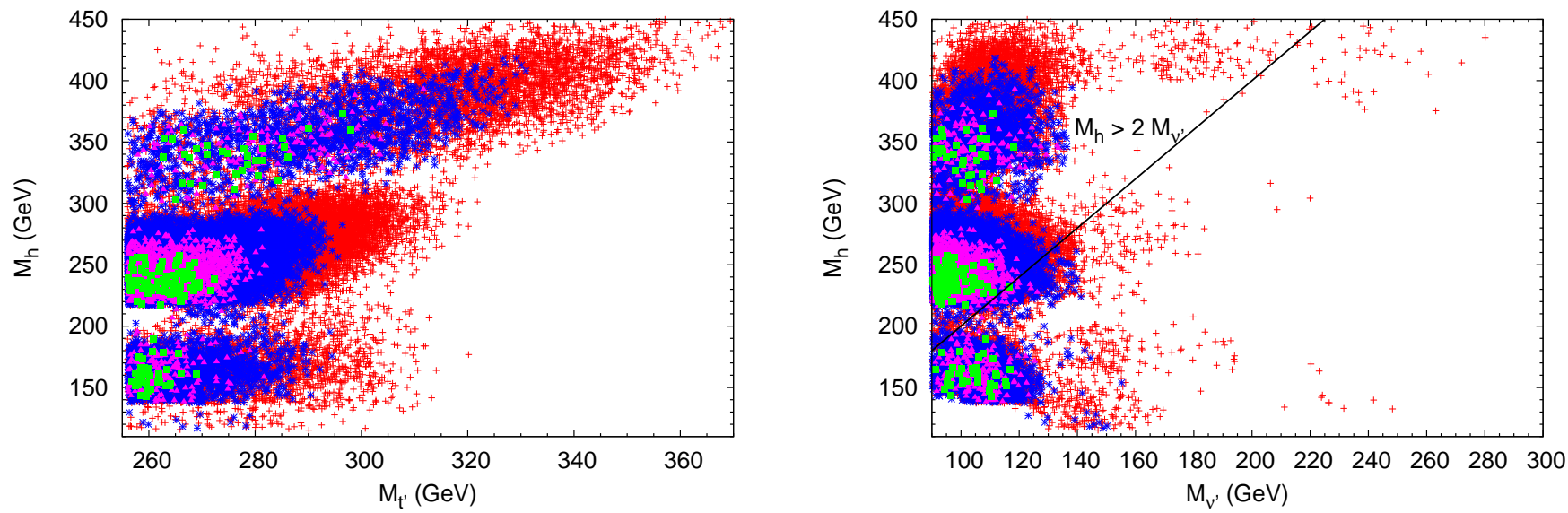

FIG. 3: Scatter plots of $M_{t^{\prime}}$ v.s. $M_{h}$ (left) and $M_{\nu^{\prime}}$ v.s. $M_{h}$ (right). The data points are the same in both figures. We took $\sin (\beta-\alpha)=1$ and varied $256 \mathrm{GeV}<M_{t^{\prime}}<552 \mathrm{GeV}, 255 \mathrm{GeV}<M_{b^{\prime}}<552 \mathrm{GeV}, 100.8 \mathrm{GeV}<M_{\tau^{\prime}}<1.23 \mathrm{TeV}, 90.3 \mathrm{GeV}<$ $M_{\nu^{\prime}}<1.23 \mathrm{TeV}, 114 \mathrm{GeV}<M_{h}<1 \mathrm{TeV}, M_{h}<M_{H}<1.5 \mathrm{TeV}, 300 \mathrm{GeV}<M_{H^{ \pm}}<1 \mathrm{TeV}, 93 \mathrm{GeV}<M_{A}<1 \mathrm{TeV}$, $0.5<\tan \beta<5$, and $\left|\lambda_{5}\right|<\pi$. The red, blue, magenta and green points correspond to the cutoff $\Lambda, 2 \mathrm{TeV} \leq \Lambda<3 \mathrm{TeV}$, $3 \mathrm{TeV} \leq \Lambda<4 \mathrm{TeV}, 4 \mathrm{TeV} \leq \Lambda<5 \mathrm{TeV}$ and $\Lambda \geq 5 \mathrm{TeV}$, respectively. All data are within the $95 \%$ C.L. limit of the $S$ and $T$ parameters.

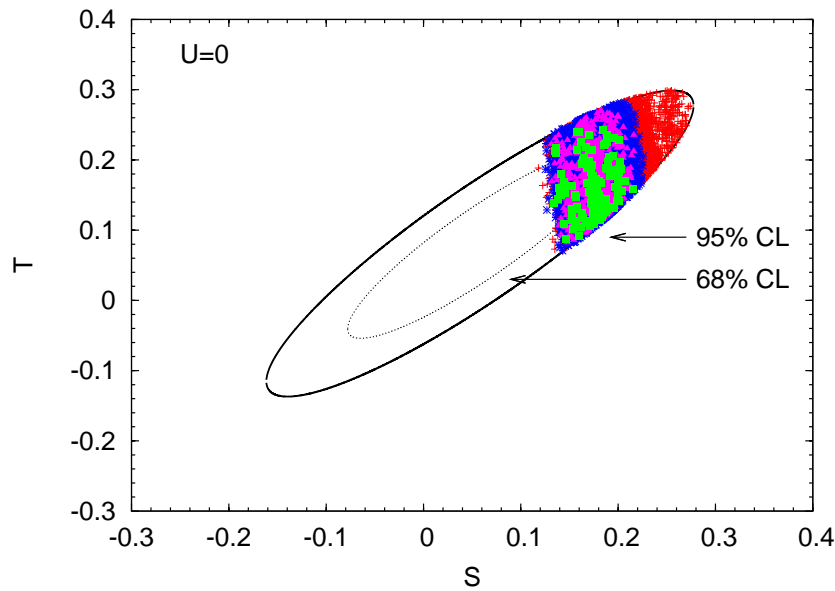

FIG. 4: The $68 \%$ and $95 \%$ C.L. constraints on the $S$ and $T$ parameters. We also showed the data points in Fig. 3.

\section{B. Methodology}

Since we assume that all parameters in the Higgs potential $V$ are real, there are eight parameters in the Higgs sector. A convenient choice is to take

$$
\begin{array}{ll}
M_{h}, \quad M_{H}, & M_{A}, \quad M_{H^{ \pm}}, \\
\lambda_{5}, & \tan \beta, \quad \sin (\beta-\alpha), \quad v(=246 \mathrm{GeV}) .
\end{array}
$$

In this paper, we fix $\sin (\beta-\alpha)=1$, at which $h$ is SM-like. Furthermore, there are four parameters corresponding to the pole masses of the fourth family quarks and leptons,

$$
M_{t^{\prime}}, \quad M_{b^{\prime}}, \quad M_{\nu^{\prime}}, \quad M_{\tau^{\prime}}
$$

Basically we search a favorable parameter space by varying the above ten parameters, as in the analysis of the SM4.

One of the problem is the matching condition between the SM4 and the THDM: At least in the decoupling limit characterized by $M_{h}^{2} \sim \mathcal{O}\left(v^{2}\right)$ and $M_{H, A, H^{ \pm}}^{2} \sim M^{2} \gg v^{2}$, we need to consider the matching of the two theories, SM4 and THDM. We here note that the structure of the yukawa sector as well as the Higgs sector is quite different in the two theories. In the THDM II, the VEV of the Higgs $\Phi_{i}(i=1,2)$ provides the fermion mass $m_{i}$ (for the fourth generation, $m_{1,2}=m_{b^{\prime}, t^{\prime}}$ or $m_{1,2}=m_{\tau^{\prime}, \nu^{\prime}}$ ), so that the relation between the mass $m_{i}$ and the yukawa coupling $y_{i}$ is

$$
m_{1}=\frac{y_{1} c_{\beta}}{\sqrt{2}} v, \quad m_{2}=\frac{y_{2} s_{\beta}}{\sqrt{2}} v
$$

where $y_{1,2}=y_{b^{\prime}, t^{\prime}}$ or $y_{1,2}=y_{\tau^{\prime}, \nu^{\prime}}$ for the fourth generation. On the other hand, the fermion mass in the SM is given by

$$
m_{i}=\frac{y_{i}^{\mathrm{SM}}}{\sqrt{2}} v, \quad(i=1,2)
$$


Besides, the running effects of the yukawa couplings are different. These affect estimate of the cutoff $\Lambda$.

A simple case is the situation $M_{H}=M_{A}=M_{H^{ \pm}}$. By definition, $M_{h}<M_{H}$ and thus we can apply the SM4 up to the scale $\mu=M_{H}=M_{A}=M_{H^{ \pm}}$, where we will identify $h$ to the SM Higgs $\phi^{0}$. Above the heavy Higgs scale, $\mu>M_{H}=M_{A}=M_{H^{ \pm}}$, we utilize the THDM description.

For a general mass spectrum of the Higgs bosons, we handle the problem as follows:

When we randomly generate data of the physical Higgs masses $M_{h, H, A, H^{ \pm}}$, we define the lightest Higgs mass among them by $\mu_{L H}$, i.e., $\mu_{L H} \equiv$ $\min \left(M_{h}, M_{H}, M_{A}, M_{H^{ \pm}}\right)$. Similarly, the second lightest one is defined by $\mu_{2 L H}$.

When $\mu_{L H}=M_{h}$, we can regard the theory in the region $\mu_{L H}<\mu<\mu_{2 L H}$ as the one Higgs doublet model. (In $\mu<\mu_{L H}$, the corresponding theory is "Higgsless".) We then improve the yukawa and Higgs quartic couplings by using the RGE's of the SM4 up to the scale $\mu_{2 L H}$. In the region $\mu>\mu_{2 L H}$, the one Higgs description can't be valid. Thus we employ the matching conditions at $\mu=\mu_{2 L H}$,

$$
\begin{aligned}
& y_{1}^{\mathrm{SM}}\left(\mu=\mu_{2 L H}\right)=y_{1}\left(\mu=\mu_{2 L H}\right) c_{\beta}, \\
& y_{2}^{\mathrm{SM}}\left(\mu=\mu_{2 L H}\right)=y_{2}\left(\mu=\mu_{2 L H}\right) s_{\beta},
\end{aligned}
$$

for the yukawa couplings, and

$$
\begin{aligned}
2 \lambda_{\mathrm{SM}}( & \left.\mu=\mu_{2 L H}\right)=\frac{M^{2}}{v^{2}} c_{\beta-\alpha}^{2} \\
& +2 \lambda_{1} s_{\alpha}^{2} c_{\beta}^{2}+2 \lambda_{2} c_{\alpha}^{2} s_{\beta}^{2}-\frac{1}{2} \lambda_{345} s_{2 \alpha} s_{2 \beta},
\end{aligned}
$$

with $\lambda_{345} \equiv \lambda_{3}+\lambda_{4}+\lambda_{5}$, for the Higgs quartic couplings, where $\lambda_{\mathrm{SM}}$ represents the SM one. Eliminating $M_{h}^{2}$ from the THDM relation (32) and using Eq. (38) instead, we obtain the quartic couplings $\lambda_{1-5}$ of the THDM at the scale $\mu=\mu_{2 L H}$. Practically, we can find $\lambda_{1-5}$ by replacing $M_{h}^{2}$ in (32) by the $\mathrm{RG}$ improved $\mathrm{SM}$ value.

On the other hand, if $\mu_{L H} \neq M_{h}$, the low energy effective theory at the $\mathrm{TeV}$ scale is no longer the SM4. In this case, we may treat the theory as the THDM from the beginning.

In this paper, we do not consider a general case with $\sin (\beta-\alpha) \neq 1$. For a full analysis of the THDM, more sophisticated prescription should be required.

\section{Numerical analysis}

The numerical analysis is similar to that in the previous section [30].
We vary the fermion masses, $256 \mathrm{GeV}<M_{t^{\prime}}<$ $552 \mathrm{GeV}, 255 \mathrm{GeV}<M_{b^{\prime}}<552 \mathrm{GeV}, 100.8 \mathrm{GeV}<$ $M_{\tau^{\prime}}<1.23 \mathrm{TeV}$ and $90.3 \mathrm{GeV}<M_{\nu^{\prime}}<1.23 \mathrm{TeV}$.

For the Higgs sector, we vary the Higgs masses, $\tan \beta$ and $\lambda_{5}$.

The Higgs quartic couplings are theoretically constrained by the stability conditions for the Higgs potential $[15]^{2}$,

$$
\begin{aligned}
& \lambda_{1}>0, \quad \lambda_{2}>0, \quad \lambda_{3}>-2 \sqrt{\lambda_{1} \lambda_{2}}, \\
& \lambda_{3}+\lambda_{4}-\left|\lambda_{5}\right|>-2 \sqrt{\lambda_{1} \lambda_{2}} .
\end{aligned}
$$

and also the tree level unitarity bounds [19, 32],

$$
\left|\tilde{a}_{ \pm}\right|,\left|\tilde{b}_{ \pm}\right|,\left|\tilde{c}_{ \pm}\right|,\left|\tilde{d}_{ \pm}\right|,\left|\tilde{e}_{1}\right|,\left|\tilde{e}_{2}\right|,\left|\tilde{f}_{ \pm}\right|,\left|\tilde{f}_{1}\right|,\left|\tilde{f}_{2}\right|<16 \pi \xi
$$

with

$$
\begin{aligned}
& \tilde{a}_{ \pm}=3\left(\lambda_{1}+\lambda_{2}\right) \pm \sqrt{9\left(\lambda_{1}-\lambda_{2}\right)^{2}+\left(2 \lambda_{3}+\lambda_{4}\right)^{2}}, \\
& \tilde{b}_{ \pm}=\left(\lambda_{1}+\lambda_{2}\right) \pm \sqrt{\left(\lambda_{1}-\lambda_{2}\right)^{2}+\lambda_{4}^{2}} \\
& \tilde{c}_{ \pm}=\tilde{d}_{ \pm}=\left(\lambda_{1}+\lambda_{2}\right) \pm \sqrt{\left(\lambda_{1}-\lambda_{2}\right)^{2}+\lambda_{5}^{2}} \\
& \tilde{e}_{1}=\lambda_{3}+2 \lambda_{4}-3 \lambda_{5} \\
& \tilde{e}_{2}=\lambda_{3}-\lambda_{5} \\
& \tilde{f}_{+}=\lambda_{3}+2 \lambda_{4}+3 \lambda_{5} \\
& \tilde{f}_{-}=\lambda_{3}+\lambda_{5} \\
& \tilde{f}_{1}=\tilde{f}_{2}=\lambda_{3}+\lambda_{4}
\end{aligned}
$$

where we take $\xi=1 / 2$, which corresponds to the radius of the Argand diagram.

Although we will ignore the mixing terms between the fourth generation and the others, the charged Higgs mass should be severely constrained by $b \rightarrow s \gamma$ and $R_{b}$, as in the three generation model [33]. In this paper, we do not fully analyze the experimental constraints. Instead, we take $M_{H^{ \pm}} \gtrsim 300 \mathrm{GeV}$ in order to evade the constraint from $b \rightarrow s \gamma[34,35]$. We also take into account the $R_{b}$-constraint for $\tan \beta<1[36,37]$. The $B^{0}-\bar{B}^{0}$ mixing yields less severer constraints only. We do not consider too small or too large $\tan \beta$, because in such a case, the yukawa couplings reach so quickly the Landau pole.

Eventually, the parameter space for the Higgs sector is taken as following; $M_{h}^{\min }<M_{h}<1 \mathrm{TeV}, M_{h}<M_{H}<$ $1.5 \mathrm{TeV}, 300 \mathrm{GeV}<M_{H^{ \pm}}<1 \mathrm{TeV}, 93 \mathrm{GeV}<M_{A}<$ $1 \mathrm{TeV}, 0.5<\tan \beta<5$, and $\left|\lambda_{5}\right|<\pi$, where $M_{h}^{\min }$ corresponds to the lower bounds of $M_{h}$ for the various values

\footnotetext{
2 The factors of the quartic couplings $\lambda_{1}$ and $\lambda_{2}$ are different from those in Refs. [15, 31, 32].
} 

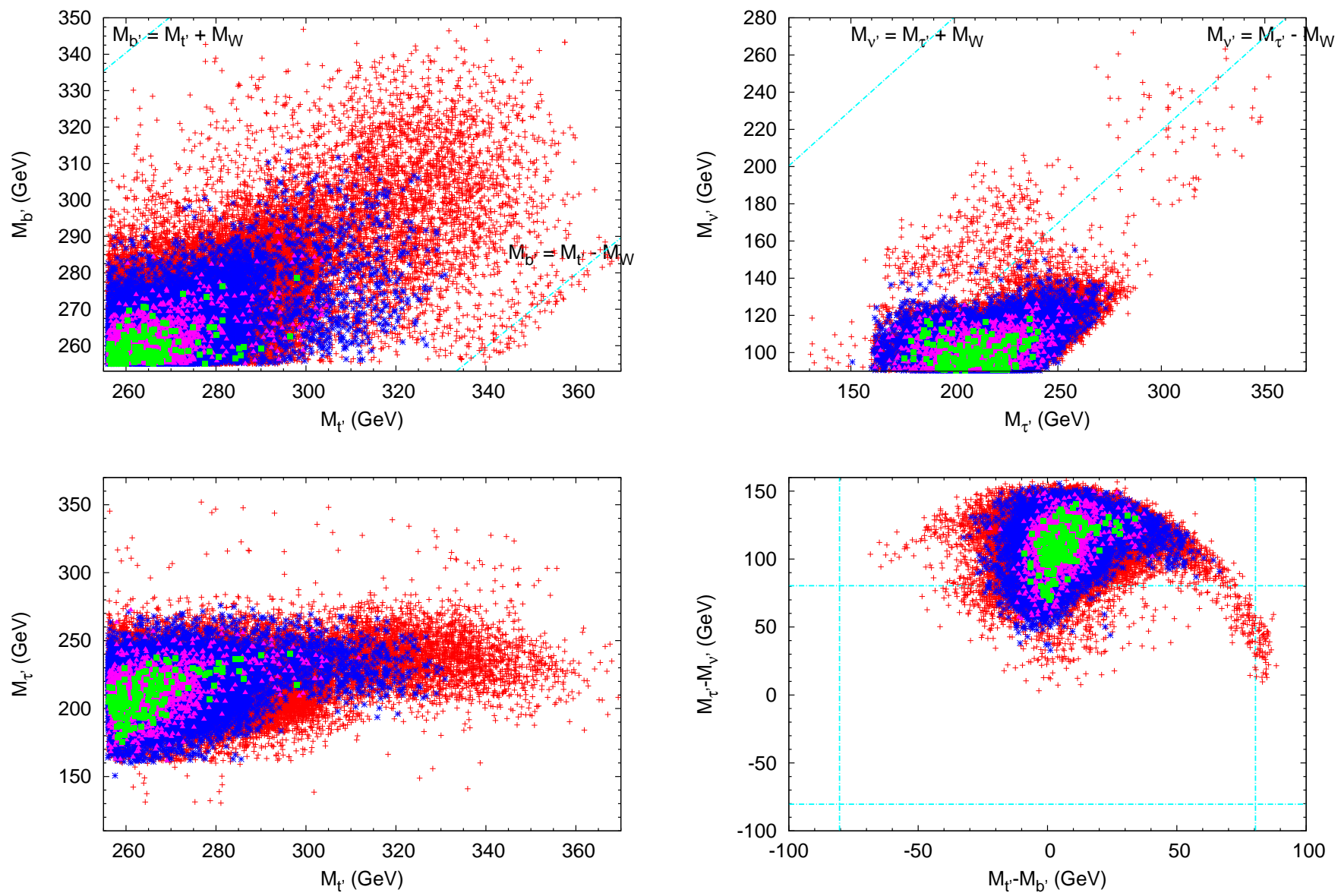

FIG. 5: $M_{t^{\prime}}$ v.s. $M_{b^{\prime}}$ (upper left), $M_{\tau^{\prime}}$ v.s. $M_{\nu^{\prime}}$ (upper right). $M_{t^{\prime}}$ v.s. $M_{\tau^{\prime}}$ (lower left) and $M_{t^{\prime}}-M_{b^{\prime}}$ v.s. $M_{\tau^{\prime}}-M_{\nu^{\prime}}$ (lower right). We took $\sin (\beta-\alpha)=1$. The data points are the same as those in Fig. 3. The blue lines in the upper figures correspond to $M_{b^{\prime}\left(\nu^{\prime}\right)}=M_{t^{\prime}\left(\tau^{\prime}\right)} \pm M_{W}$. The blue lines in the lower right correspond to $\pm M_{W}$.

of $\sin ^{2}(\beta-\alpha)$ which can be read from the constraints of the LEP experiments [38]. In the case of $\sin (\beta-\alpha)=1$, it corresponds to the SM bound, $M_{h}^{\min }=114 \mathrm{GeV}$.

Taking into account the RGE's for the yukawa and Higgs quartic couplings [31, 39], which are shown in Appendix B, and also imposing the instability bounds for the Higgs potential and the perturbative unitarity bounds on the yukawa and Higgs quartic couplings, we calculate the cutoff $\Lambda$ at which some new physics or strong dynamics enters. The masses of the fermions and the Higgs bosons are also constrained by the $(S, T)$ parameters [3].

Since the parameter space is enormous, we need to find efficiently the cutoff $\Lambda$ unlike in the analysis of the SM4. After generating the primary data with an equal probability, we refine all parameters so as to make $\Lambda$ larger. We cannot deny the possibility that we may overlook some favorable parameter region, if the primary data might be too rough.

We depict the results in Figs. 3-7.

The relation between $M_{t^{\prime}\left(\nu^{\prime}\right)}$ and $M_{h}$ is shown in Fig. 3 . All data points are within $95 \%$ C.L. limit of the $(S, T)$ parameters. (See Fig. 4.) Similarly, the mass relations between the fourth generation fermions are depicted in Fig. 5. The masses of the extra heavy Higgs bosons are described in Fig. 6. The allowed parameter region for $M_{H^{ \pm}}$and $\tan \beta$ is shown in the left of Fig. 7. The values of $\lambda_{5}$ can be read from the right of Fig. 7 by using Eq. (32).

It is noticeable that the decay channels $h \rightarrow \bar{\nu}^{\prime} \nu^{\prime}$ and $\tau^{\prime} \rightarrow \nu^{\prime}+W^{-}$are allowed in a wide parameter range. (See the right of Fig. 3 and the lower right of Fig. 5.) Notice that $\tan \beta \simeq 1$ in order to relax the appearance of the Landau pole for the yukawa couplings. (See the left 


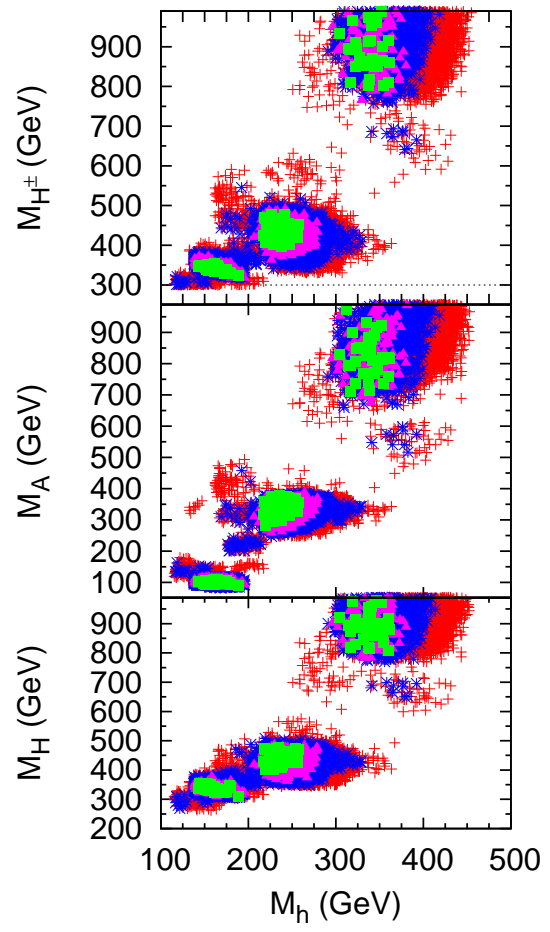

FIG. 6: Masses of the extra Higgs bosons. We took $\sin (\beta-$ $\alpha)=1$. The data points are the same as those in Fig. 3 .

of Fig. 7.)

Schematically speaking, as shown in the left of Fig. 3 and Fig. 6, the results consist of high and low $M_{h}$ regimes, $M_{h} \gtrsim M_{t^{\prime}}$ and $M_{h} \lesssim M_{t^{\prime}}$, respectively, where we took the cutoff $\Lambda \gtrsim 2 \mathrm{TeV}$.

We can confirm that the decoupling regime of the extra Higgs bosons, say, $M_{H} \sim M_{H^{ \pm}} \gtrsim 800 \mathrm{GeV}$ and $M_{A} \gtrsim$ $700 \mathrm{GeV}$, is contained in the region $M_{h} \gtrsim M_{t^{\prime}}$. (See Fig. 6 and the right of Fig. 7.) This is consistent with the analysis of the SM4. In this case, the extra heavy Higgs bosons can decay into a quark/lepton pair of the fourth generation, if kinematically allowed.

A new feature of the two Higgs extension is thus characterized by the low $M_{h}$ regime, $M_{h} \lesssim M_{t^{\prime}}$. In the $(S, T)$ analysis, this regime is more favorable than the high $M_{h}$ one, i.e., most of the data points inside the $68 \%$ C.L. limit of the $(S, T)$-constraints are for the former. The point is that even for the low $M_{h}$, the Higgs potential can be stable owing to the dynamics of the Higgs quartic couplings. When we take into account the Tevatron bounds of the fourth generation quark masses, $M_{t^{\prime}}>311 \mathrm{GeV}$ and $M_{b^{\prime}}>338 \mathrm{GeV}$ [28], only a small parameter space is left, however. Nevertheless, we here mention that the parameter region with $M_{h} \approx 100-300 \mathrm{GeV}$ is interesting, because the extra Higgs masses can be almost degenerate, $M_{H} \sim M_{H^{ \pm}} \sim M_{A} \sim 300-400 \mathrm{GeV}$, and also a scenario with $M^{2}=0$ is possible (see the right of Fig. 7). We also note that even in this regime, the leptonic decays of the Higgs bosons such as $h \rightarrow \bar{\nu}^{\prime} \nu^{\prime}, H^{-} \rightarrow \bar{\nu}^{\prime} \tau^{\prime}$ and etc. are open in a certain parameter space.

We have analyzed only the case of $\sin (\beta-\alpha)=1$. If we extend our analysis with a general $\sin (\beta-\alpha)$, more favorable and exotic Higgs mass spectra can be found. This will be performed elsewhere.

\section{SUMMARY AND DISCUSSIONS}

We have reanalyzed the constraints on the mass spectrum of the fourth generation fermions and the Higgs bosons for the SM4 and the THDM II with $\sin (\beta-\alpha)=1$. We showed that there are the noticeable correlations among the mass spectrum of the fermions and the Higgs bosons.

For the SM4, the favorable mass range of the physical Higgs boson $\phi^{0}$ is $M_{\phi^{0}} \gtrsim M_{t^{\prime}}\left(M_{\phi^{0}} \gtrsim M_{t^{\prime}}-50 \mathrm{GeV}\right)$ for the cutoff $\Lambda \geq 2 \mathrm{TeV}(\Lambda \geq 1 \mathrm{TeV})$. We also found that the favorable parameter space is mainly contained in the region,

$$
\begin{aligned}
& \frac{3\left(M_{t^{\prime}}-M_{b^{\prime}}\right)^{2}}{M_{W}^{2}}+\frac{\left(M_{\tau^{\prime}}-M_{\nu^{\prime}}\right)^{2}}{M_{W}^{2}} \\
& \approx(1.3-2.0)+1.4 \ln \frac{M_{\phi^{0}}}{M_{\phi^{0}, \text { ref }}},
\end{aligned}
$$

and

$$
M_{t^{\prime}}>M_{b^{\prime}}, \quad M_{\tau^{\prime}}>M_{\nu^{\prime}} .
$$

(See also the semicircles in lower right of Fig. 2.) We showed the data samples corresponding to several scenarios in Table I.

For the THDM II with $\sin (\beta-\alpha)=1$, schematically speaking, there are two domains for the favorable mass range of the light $\mathrm{CP}$ even Higgs $h, M_{h} \gtrsim M_{t^{\prime}}$ and $M_{h} \lesssim M_{t^{\prime}}$. The extra heavy Higgs decoupling regime is contained in the former. This is consistent with the analysis of the SM4. On the other hand, an almost degenerate Higgs mass spectrum such as $M_{h} \approx 100-300 \mathrm{GeV}$ and $M_{H} \sim M_{H^{ \pm}} \sim M_{A} \sim 300-400 \mathrm{GeV}$ is allowed in the latter and in a part of the former. In this case, a model with $M^{2}=0$ is not excluded. (See the right of Fig. 7.) As for the value of $\tan \beta$, we found that $\tan \beta \approx 1$ is favorable in the both domains.

Concerning the decay channels of the charged leptons, we found that $\tau^{\prime} \rightarrow \nu^{\prime}+W^{-}$is allowed in a wide parameter space in the SM4 and the THDM II. (See the lower right of Figs. 2 and 5.) The fourth generation quark masses can be degenerate $M_{t^{\prime}}=M_{b^{\prime}}$ or the decay channel $t^{\prime} \rightarrow b^{\prime}+W^{(*)}$ can be open, depending on the mass 

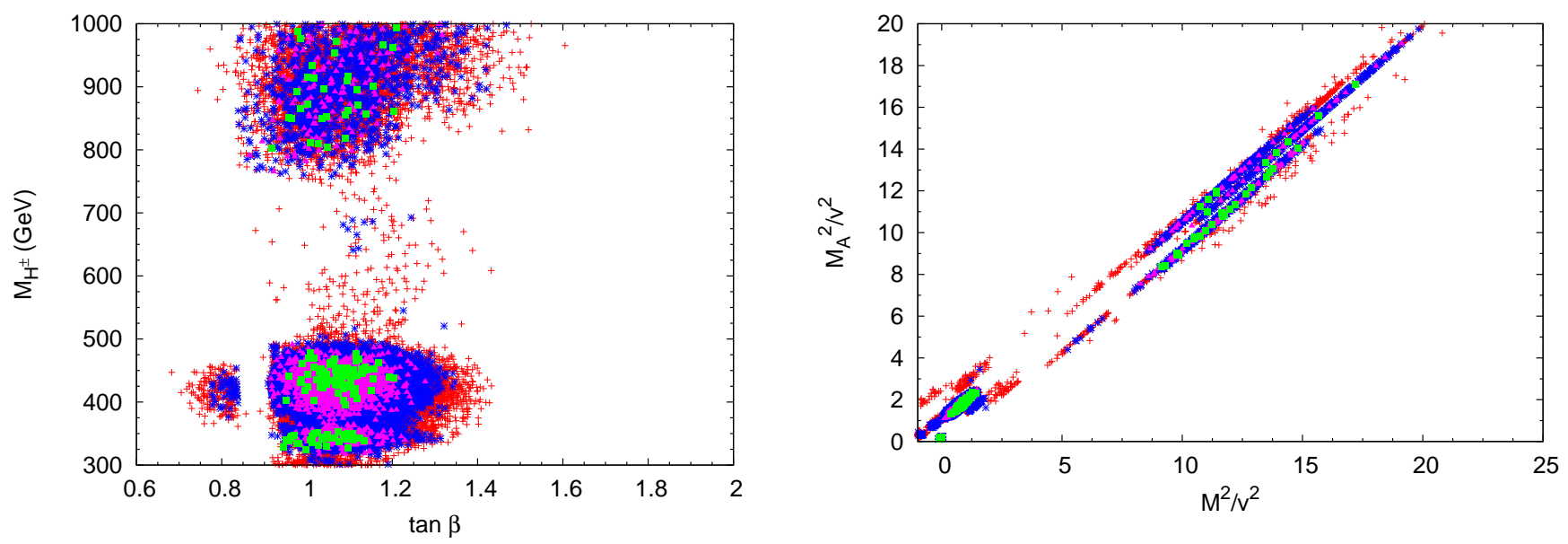

FIG. 7: $\tan \beta$ v.s. $M_{H^{ \pm}}$and $M^{2} / v^{2}$ v.s. $M_{A}^{2} / v^{2}$. We took $\sin (\beta-\alpha)=1$. The data points are the same as those in Fig. 3 . We can read the value of $\lambda_{5}$ from the right figure by using Eq. (32).

difference $M_{\tau^{\prime}}-M_{\nu^{\prime}}$. (See the lower right of Figs. 2 and 5.) The Higgs $\phi^{0}$ in the SM4 and the light CP even Higgs $h$ in the THDM II can decay into a pair of the fourth generation neutrinos. (See the right of Figs. 1 and 3.) Furthermore, in the THDM II, a scenario that all Higgs bosons decay into a pair of the fourth generation leptons is possible, i.e., $h \rightarrow \bar{\nu}^{\prime} \nu^{\prime}$ and $H^{-} \rightarrow \bar{\nu}^{\prime} \tau^{\prime}$, etc.. (For studies of collider signals of the fourth generation leptons, see, e.g., Ref. [40].) We also comment that the main decay channel of the heavy $\mathrm{CP}$ even Higgs $H$ can be $H \rightarrow \bar{t}^{\prime} t^{\prime}, \bar{b}^{\prime} b^{\prime}$, if kinematically allowed [41]. Thus the phenomenology of the fourth generation models is very rich.

The implications of the analysis in this paper are obvious: If the Tevatron and/or LHC discover $t^{\prime}$ and/or $b^{\prime}$, the Higgs mass spectrum will be suggested, depending on the models. On the other hand, if the LHC excludes the $t^{\prime}$ and/or $b^{\prime}$ masses $M_{t^{\prime}, b^{\prime}} \lesssim 500 \mathrm{GeV}$ at early stage, a big parameter space will be gone. In this case, essentially, a nonperturbative regime will be left to be examined.

Many issues remain to be explored:

- We did not consider a general case of $\sin (\beta-\alpha)$. There probably exist more favorable and exotic parameter regions in the THDM II. Moreover, we may consider a different yukawa structure other than the type II [42].

- Majorana neutrinos can reduce $S$ and $T$ [43]. It may affect the mass spectrum of the fourth generation fermions and the Higgs bosons.

- The two-loop effects are probably relevant for more precise predictions of the mass spectrum. The the- oretical lower bound for the Higgs mass, which essentially corresponds to the instability bound of the Higgs potential, will be almost unchanged, however, because the parameters certainly stay in a perturbative region.

- We did not take into account the mixing angle between the fourth and third generations. This is, of course, very important to discuss the flavor constraints and realistic decay chains of the fourth generation quarks and leptons [44, 45].

- If the main branching ratios of $t^{\prime}$ and $b^{\prime}$ are different from well-studied ones in experiments, a first evidence of the fourth generation might be found in the Higgs physics, for example, as a huge enhancement of the golden mode, $g g \rightarrow \phi^{0} / h \rightarrow Z Z$. Concerning the loop induced processes, it is important to notice that the loop effects in $h \rightarrow g g, h \rightarrow \gamma \gamma$ and $A \rightarrow g g, \gamma \gamma$ are quite different [10]. Related to such possibilities, there should exist very large non-decoupling effects in the triple Higgs coupling arising from the fourth generation quarks and leptons $[32,46]$. The triple Higgs coupling is testable at the LHC/vLHC/sLHC [47] and at the ILC [48].

- The fourth generation model may play an important role in B-CP asymmetries $[49,50]$ and also in the electroweak baryogenesis [51].

- Last but not least, if only a nonperturbative regime is left in the near future, what kind of study will be relevant? For example, when the fourth generation quarks are ultraheavy beyond the perturbative uni- 
tarity bound, is there some nonperturbative effect in the nonresonant $g g \rightarrow Z Z$ process [52]?

\section{Acknowledgments}

The author thanks to S. Kanemura for fruitful discussions and to V.A.Miransky for useful comments. This work is supported by the Grant-in-Aid for Science Research, Ministry of Education, Culture, Sports, Science and Technology, Japan, No. 16081211.

\section{Appendix A: RGE's for the SM4}

For the gauge couplings, the RGE's are

$$
\left(16 \pi^{2}\right) \mu \frac{\partial}{\partial \mu} g_{i}=-c_{i} g_{i}^{3},
$$

with

$$
\begin{aligned}
& c_{1}=-\frac{1}{6} N_{H}-\frac{20}{9} N_{g}, \\
& c_{2}=\frac{22}{3}-\frac{4}{3} N_{g}-\frac{1}{6} N_{H}, \\
& c_{3}=11-\frac{4}{3} N_{g},
\end{aligned}
$$

where $N_{g}$ and $N_{H}$ denote the number of generations and the number of Higgs doublets, respectively. Although we did not show explicitly the formulae, we take into account the threshold effects.

The RGE's of the yukawa couplings are [22, 23]

$$
\begin{gathered}
\left(16 \pi^{2}\right) \mu \frac{\partial}{\partial \mu} y_{t}=-\left(8 g_{3}^{2}+\frac{9}{4} g_{2}^{2}+\frac{17}{12} g_{1}^{2}\right) y_{t}+\frac{9}{2} y_{t}^{3} \\
+y_{t}\left[3 y_{t^{\prime}}^{2}+3 y_{b^{\prime}}^{2}+\frac{3}{2} y_{b}^{2}+y_{\nu^{\prime}}^{2}+y_{\tau^{\prime}}^{2}+y_{\tau}^{2}\right], \quad(\mathrm{A} 5 \\
\left(16 \pi^{2}\right) \mu \frac{\partial}{\partial \mu} y_{b}=-\left(8 g_{3}^{2}+\frac{9}{4} g_{2}^{2}+\frac{5}{12} g_{1}^{2}\right) y_{b}+\frac{9}{2} y_{b}^{3} \\
+y_{b}\left[3 y_{b^{\prime}}^{2}+3 y_{t^{\prime}}^{2}+\frac{3}{2} y_{t}^{2}+y_{\nu^{\prime}}^{2}+y_{\tau^{\prime}}^{2}+y_{\tau}^{2}\right], \quad(\mathrm{A} 5 \mathrm{~g}) \\
\left(16 \pi^{2}\right) \mu \frac{\partial}{\partial \mu} y_{\tau}=-\left(\frac{9}{4} g_{2}^{2}+\frac{15}{4} g_{1}^{2}\right) y_{\tau}+\frac{5}{2} y_{\tau}^{3} \\
+y_{\tau}\left[3 y_{t^{\prime}}^{2}+3 y_{b^{\prime}}^{2}+3 y_{t}^{2}+3 y_{b}^{2}+y_{\nu^{\prime}}^{2}+y_{\tau^{\prime}}^{2}\right],(\mathrm{A} \\
\left(16 \pi^{2}\right) \mu \frac{\partial}{\partial \mu} y_{t^{\prime}}=-\left(8 g_{3}^{2}+\frac{9}{4} g_{2}^{2}+\frac{17}{12} g_{1}^{2}\right) y_{t^{\prime}}+\frac{9}{2} y_{t^{\prime}}^{3} \\
+y_{t^{\prime}}\left[\frac{3}{2} y_{b^{\prime}}^{2}+3 y_{t}^{2}+3 y_{b}^{2}+y_{\nu^{\prime}}^{2}+y_{\tau^{\prime}}^{2}+y_{\tau}^{2}\right], \quad(\mathrm{A} 5 \mathrm{~d})
\end{gathered}
$$

$$
\begin{gathered}
\left(16 \pi^{2}\right) \mu \frac{\partial}{\partial \mu} y_{b^{\prime}}=-\left(8 g_{3}^{2}+\frac{9}{4} g_{2}^{2}+\frac{5}{12} g_{1}^{2}\right) y_{b^{\prime}}+\frac{9}{2} y_{b^{\prime}}^{3} \\
+y_{b^{\prime}}\left[\frac{3}{2} y_{t^{\prime}}^{2}+3 y_{t}^{2}+3 y_{b}^{2}+y_{\nu^{\prime}}^{2}+y_{\tau^{\prime}}^{2}+y_{\tau}^{2}\right], \\
\left(16 \pi^{2}\right) \mu \frac{\partial}{\partial \mu} y_{\nu^{\prime}}=-\left(\frac{9}{4} g_{2}^{2}+\frac{3}{4} g_{1}^{2}\right) y_{\nu^{\prime}}+\frac{5}{2} y_{\nu^{\prime}}^{3} \\
+y_{\nu^{\prime}}\left[3 y_{t^{\prime}}^{2}+3 y_{b^{\prime}}^{2}+3 y_{t}^{2}+3 y_{b}^{2}-\frac{1}{2} y_{\tau^{\prime}}^{2}+y_{\tau}^{2}\right],(\mathrm{A} \\
\left(16 \pi^{2}\right) \mu \frac{\partial}{\partial \mu} y_{\tau^{\prime}}=-\left(\frac{9}{4} g_{2}^{2}+\frac{15}{4} g_{1}^{2}\right) y_{\tau^{\prime}}+\frac{5}{2} y_{\tau^{\prime}}^{3} \\
+y_{\tau^{\prime}}\left[3 y_{t^{\prime}}^{2}+3 y_{b^{\prime}}^{2}+3 y_{t}^{2}+3 y_{b}^{2}-\frac{1}{2} y_{\nu^{\prime}}^{2}+y_{\tau}^{2}\right],(\mathrm{A}
\end{gathered}
$$

where we did not show explicitly the threshold effects of the fermions. Inside of the square bracket, $y_{f}^{2}(f=$ $\left.t^{\prime}, b^{\prime}, \cdots\right)$ should be regarded as $y_{f}^{2} \theta\left(\mu-m_{f}\right)$, where $m_{f}$ is the corresponding $\overline{\mathrm{MS}}$-mass of the fermions.

The RGE for the Higgs quartic coupling is given by

$$
\begin{aligned}
& \left(16 \pi^{2}\right) \mu \frac{\partial}{\partial \mu} \lambda=24 \lambda^{2}-3 \lambda\left(3 g_{2}^{2}+g_{1}^{2}\right) \\
& +4 \lambda\left[3\left(y_{t^{\prime}}^{2}+y_{b^{\prime}}^{2}+y_{t}^{2}+y_{b}^{2}\right)+y_{\tau^{\prime}}^{2}+y_{\nu^{\prime}}^{2}+y_{\tau}^{2}\right] \\
& \quad-2\left[3\left(y_{t^{\prime}}^{4}+y_{b^{\prime}}^{4}+y_{t}^{4}+y_{b}^{4}\right)+y_{\tau^{\prime}}^{4}+y_{\nu^{\prime}}^{4}+y_{\tau}^{4}\right] \\
& +\frac{3}{8}\left[2 g_{2}^{4}+\left(g_{2}^{2}+g_{1}^{2}\right)^{2}\right] .
\end{aligned}
$$

\section{Appendix B: RGE's for the THDM II}

Let us consider the RGE's for the THDM II.

The RGE's for Yukawa couplings are given by $[31,39]$

$$
\begin{gathered}
\left(16 \pi^{2}\right) \mu \frac{\partial}{\partial \mu} y_{t}=-\left(8 g_{3}^{2}+\frac{9}{4} g_{2}^{2}+\frac{17}{12} g_{1}^{2}\right) y_{t}+\frac{9}{2} y_{t}^{3} \\
+y_{t}\left[3 y_{t^{\prime}}^{2}+\frac{1}{2} y_{b}^{2}+y_{\nu^{\prime}}^{2}\right], \\
\left(16 \pi^{2}\right) \mu \frac{\partial}{\partial \mu} y_{b}=-\left(8 g_{3}^{2}+\frac{9}{4} g_{2}^{2}+\frac{5}{12} g_{1}^{2}\right) y_{b}+\frac{9}{2} y_{b}^{3} \\
+y_{b}\left[3 y_{b^{\prime}}^{2}+\frac{1}{2} y_{t}^{2}+y_{\tau^{\prime}}^{2}+y_{\tau}^{2}\right], \quad(\mathrm{B} 1 b) \\
\left(16 \pi^{2}\right) \mu \frac{\partial}{\partial \mu} y_{\tau}=-\left(\frac{9}{4} g_{2}^{2}+\frac{15}{4} g_{1}^{2}\right) y_{\tau}+\frac{5}{2} y_{\tau}^{3} \\
+y_{\tau}\left[3 y_{b}^{2}+3 y_{b^{\prime}}^{2}+y_{\tau^{\prime}}^{2}\right]
\end{gathered}
$$




$$
\begin{gathered}
\left(16 \pi^{2}\right) \mu \frac{\partial}{\partial \mu} y_{t^{\prime}}=-\left(8 g_{3}^{2}+\frac{9}{4} g_{2}^{2}+\frac{17}{12} g_{1}^{2}\right) y_{t^{\prime}}+\frac{9}{2} y_{t^{\prime}}^{3} \\
+y_{t^{\prime}}\left[3 y_{t}^{2}+\frac{1}{2} y_{b^{\prime}}^{2}+y_{\nu^{\prime}}^{2}\right], \\
\left(16 \pi^{2}\right) \mu \frac{\partial}{\partial \mu} y_{b^{\prime}}=-\left(8 g_{3}^{2}+\frac{9}{4} g_{2}^{2}+\frac{5}{12} g_{1}^{2}\right) y_{b^{\prime}}+\frac{9}{2} y_{b^{\prime}}^{3} \\
+y_{b^{\prime}}\left[3 y_{b}^{2}+\frac{1}{2} y_{t^{\prime}}^{2}+y_{\tau^{\prime}}^{2}+y_{\tau}^{2}\right], \quad(\mathrm{B} 1 \\
\left(16 \pi^{2}\right) \mu \frac{\partial}{\partial \mu} y_{\nu^{\prime}}=-\left(\frac{9}{4} g_{2}^{2}+\frac{3}{4} g_{1}^{2}\right) y_{\nu^{\prime}}+\frac{5}{2} y_{\nu^{\prime}}^{3} \\
+y_{\nu^{\prime}}\left[3 y_{t}^{2}+3 y_{t^{\prime}}^{2}+\frac{1}{2} y_{\tau^{\prime}}^{2}\right], \\
\left.+16 \pi^{2}\right) \mu \frac{\partial}{\partial \mu} y_{\tau^{\prime}}=-\left(\frac{9}{4} g_{2}^{2}+\frac{15}{4} g_{1}^{2}\right) y_{\tau^{\prime}}+\frac{5}{2} y_{\tau^{\prime}}^{3} \\
+y_{\tau^{\prime}}\left[3 y_{b}^{2}+3 y_{b^{\prime}}^{2}+\frac{1}{2} y_{\nu^{\prime}}^{2}+y_{\tau}^{2}\right]
\end{gathered}
$$

where we ignored $y_{c}, y_{\nu}$, etc..

The RGE's for the Higgs quartic self-couplings are [31, $39]$

$$
\begin{aligned}
&\left(16 \pi^{2}\right) \mu \frac{\partial}{\partial \mu} \lambda_{1}=24 \lambda_{1}^{2}+2 \lambda_{3}^{2}+2 \lambda_{3} \lambda_{4}+\lambda_{4}^{2}+\lambda_{5}^{2} \\
&-3 \lambda_{1}\left(3 g_{2}^{2}+g_{1}^{2}\right)+\frac{3}{8}\left[2 g_{2}^{4}+\left(g_{2}^{2}+g_{1}^{2}\right)^{2}\right] \\
&+4 \lambda_{1}\left[3 y_{b^{\prime}}^{2}+3 y_{b}^{2}+y_{\tau^{\prime}}^{2}+y_{\tau}^{2}\right] \\
&-2\left[3 y_{b^{\prime}}^{4}+3 y_{b}^{4}+y_{\tau^{\prime}}^{4}+y_{\tau}^{4}\right], \\
&\left(16 \pi^{2}\right) \mu \frac{\partial}{\partial \mu} \lambda_{2}=24 \lambda_{2}^{2}+2 \lambda_{3}^{2}+2 \lambda_{3} \lambda_{4}+\lambda_{4}^{2}+\lambda_{5}^{2} \\
&-3 \lambda_{2}\left(3 g_{2}^{2}+g_{1}^{2}\right)+\frac{3}{8}\left[2 g_{2}^{4}+\left(g_{2}^{2}+g_{1}^{2}\right)^{2}\right] \\
&+4 \lambda_{2}\left[3 y_{t^{\prime}}^{2}+3 y_{t}^{2}+y_{\nu^{\prime}}^{2}\right] \\
&-2\left[3 y_{t^{\prime}}^{4}+3 y_{t}^{4}+y_{\nu^{\prime}}^{4}\right]
\end{aligned}
$$

$$
\begin{gathered}
\left(16 \pi^{2}\right) \mu \frac{\partial}{\partial \mu} \lambda_{3}=2\left(\lambda_{1}+\lambda_{2}\right)\left(6 \lambda_{3}+2 \lambda_{4}\right)+4 \lambda_{3}^{2}+2 \lambda_{4}^{2} \\
+2 \lambda_{5}^{2}-3 \lambda_{3}\left(3 g_{2}^{2}+g_{1}^{2}\right)+\frac{3}{4}\left[2 g_{2}^{4}+\left(g_{2}^{2}-g_{1}^{2}\right)^{2}\right] \\
+2 \lambda_{3}\left[3\left(y_{t}^{2}+y_{b}^{2}+y_{t^{\prime}}^{2}+y_{b^{\prime}}^{2}\right)+y_{\nu^{\prime}}^{2}+y_{\tau^{\prime}}^{2}+y_{\tau}^{2}\right] \\
-4\left[3 y_{t^{\prime}}^{2} y_{b^{\prime}}^{2}+3 y_{t}^{2} y_{b}^{2}+y_{\nu^{\prime}}^{2} y_{\tau^{\prime}}^{2}\right], \\
\left(16 \pi^{2}\right) \mu \frac{\partial}{\partial \mu} \lambda_{4}=4\left(\lambda_{1}+\lambda_{2}+2 \lambda_{3}+\lambda_{4}\right) \lambda_{4}+8 \lambda_{5}^{2} \\
-3 \lambda_{4}\left(3 g_{2}^{2}+g_{1}^{2}\right)+3 g_{1}^{2} g_{2}^{2} \\
+2 \lambda_{4}\left[3\left(y_{t}^{2}+y_{b}^{2}+y_{t^{\prime}}^{2}+y_{b^{\prime}}^{2}\right)+y_{\nu^{\prime}}^{2}+y_{\tau^{\prime}}^{2}+y_{\tau}^{2}\right] \\
+4\left[3 y_{t^{\prime}}^{2} y_{b^{\prime}}^{2}+3 y_{t}^{2} y_{b}^{2}+y_{\nu^{\prime}}^{2} y_{\tau^{\prime}}^{2}\right],
\end{gathered}
$$

$$
\begin{gathered}
\left(16 \pi^{2}\right) \mu \frac{\partial}{\partial \mu} \lambda_{5}=\lambda_{5}\left[4\left(\lambda_{1}+\lambda_{2}\right)+8 \lambda_{3}+12 \lambda_{4}\right. \\
-3\left(3 g_{2}^{2}+g_{1}^{2}\right)+2\left\{3\left(y_{t}^{2}+y_{b}^{2}+y_{t^{\prime}}^{2}+y_{b^{\prime}}^{2}\right)\right. \\
\left.\left.+y_{\nu^{\prime}}^{2}+y_{\tau^{\prime}}^{2}+y_{\tau}^{2}\right\}\right]
\end{gathered}
$$

Note that the definitions for $\lambda_{1}$ and $\lambda_{2}$ are twice larger than those in Ref. [31].
[1] For a comprehensive review, see, e.g., P. H. Frampton, P. Q. Hung and M. Sher, Phys. Rept. 330, 263 (2000).

[2] For a recent overview, see, e.g., B. Holdom, W. S. Hou,
T. Hurth, M. L. Mangano, S. Sultansoy and G. Unel, PMC Phys. A 3, 4 (2009) [arXiv:0904.4698 [hep-ph]]. [3] H. J. He, N. Polonsky and S. f. Su, Phys. Rev. D 64, 
053004 (2001).

[4] V. A. Novikov, L. B. Okun, A. N. Rozanov and M. I. Vysotsky, JETP Lett. 76, 127 (2002) [Pisma Zh. Eksp. Teor. Fiz. 76, 158 (2002)] [arXiv:hep-ph/ 0203132]; V. A. Novikov, A. N. Rozanov and M. I. Vysotsky, arXiv:0904.4570 [hep-ph].

[5] G. D. Kribs, T. Plehn, M. Spannowsky and T. M. P. Tait, Phys. Rev. D 76, 075016 (2007) [arXiv:0706.3718 [hep$\mathrm{ph}]$.

[6] J. A. Aguilar-Saavedra, Phys. Lett. B 625, 234 (2005) [Erratum-ibid. B 633, 792 (2006)]; O. Cakir, H. Duran Yildiz, R. Mehdiyev and I. Turk Cakir, Eur. Phys. J. C 56, 537 (2008) [arXiv:0801.0236 [hep-ph]]; V. E. Ozcan, S. Sultansoy and G. Unel, arXiv:0802.2621 [hepex]; V. E. Ozcan, S. Sultansoy and G. Unel, Eur. Phys. J. C 57 (2008) 621; E. L. Berger and Q. H. Cao, arXiv: 0909.3555 [hep-ph].

[7] B. Holdom, Phys. Rev. Lett. 57, 2496 (1986) [Erratumibid. 58, 177 (1987)]; C. T. Hill, M. A. Luty and E. A. Paschos, Phys. Rev. D 43, 3011 (1991); B. Holdom, Phys. Rev. D 54, 721 (1996); JHEP 0608, 076 (2006).

[8] M. Hashimoto and V. A. Miransky, Phys. Rev. D 80, 013004 (2009) [arXiv:0901.4354 [hep-ph]].

[9] M. Hashimoto and V. A. Miransky, arXiv:0912.4453 [hep-ph].

[10] For a comprehensive review, see, J. F. Gunion, H. E. Haber, G. Kane, and S. Dawson, The Higgs Hunter's Guide (Perseus Publishing, Cambridge, MA, 1990).

[11] See, e.g., J. F. Gunion and H. E. Haber, Phys. Rev. D 67, 075019 (2003).

[12] C. T. Hill, Phys. Lett. B 266, 419 (1991); C. T. Hill, Phys. Lett. B 345, 483 (1995); For a recent comprehensive review, see, e.g., C. T. Hill and E. H. Simmons, Phys. Rept. 381, 235 (2003) [Erratum-ibid. 390, 553 (2004)].

[13] W. A. Bardeen, C. T. Hill and M. Lindner, Phys. Rev. D 41, 1647 (1990).

[14] M. A. Luty, Phys. Rev. D 41, 2893 (1990).

[15] N. G. Deshpande and E. Ma, Phys. Rev. D 18, 2574 (1978).

[16] For a review, see, M. Sher, Phys. Rept. 179, 273 (1989).

[17] M. S. Chanowitz, M. A. Furman and I. Hinchliffe, Phys. Lett. B 78, 285 (1978); Nucl. Phys. B 153, 402 (1979).

[18] B. W. Lee, C. Quigg and H. B. Thacker, Phys. Rev. Lett. 38, 883 (1977); Phys. Rev. D 16, 1519 (1977).

[19] S. Kanemura, T. Kubota and E. Takasugi, Phys. Lett. B 313, 155 (1993) [arXiv:hep-ph/9303263].

[20] A. G. Akeroyd, A. Arhrib and E. M. Naimi, Phys. Lett. B 490, 119 (2000) [arXiv:hep-ph/0006035].

[21] M. E. Peskin and T. Takeuchi, Phys. Rev. Lett. 65, 964 (1990); Phys. Rev. D 46, 381 (1992).

[22] For a general discussion including the two-loop effects, see, e.g., M. E. Machacek and M. T. Vaughn, Nucl. Phys. B 222, 83 (1983); Nucl. Phys. B 236, 221 (1984); Nucl. Phys. B 249, 70 (1985).

[23] There has been numerous works on this subject, for example, C. T. Hill, Phys. Rev. D 24, 691 (1981); C. Wetterich, Phys. Lett. B 104, 269 (1981); M. Tanimoto, T. Hayashi, R. Najima and S. Wakaizumi, Prog. Theor.
Phys. 76, 1098 (1986); H. B. Nielsen, A. V. Novikov, V. A. Novikov and M. I. Vysotsky, Phys. Lett. B 374, 127 (1996) [arXiv:hep-ph/9511340]; D. Dooling, K. Kang and S. K. Kang, Int. J. Mod. Phys. A 14, 1605 (1999) [arXiv:hep-ph/9710258]; P. Q. Hung, Phys. Rev. Lett. 80, 3000 (1998) [arXiv:hep-ph/9712338]; Yu. F. Pirogov and O. V. Zenin, Eur. Phys. J. C 10, 629 (1999) [arXiv: hep-ph/9808396].

[24] C. Amsler et al. [Particle Data Group], Phys. Lett. B 667, 1 (2008).

[25] [ALEPH Collaboration and DELPHI Collaboration and L3 Collaboration and ], Phys. Rept. 427, 257 (2006) [arXiv:hep-ex/0509008].

[26] [ALEPH Collaboration and CDF Collaboration and D0 Collaboration and an], arXiv:0811.4682 [hep-ex]; http: //lepewwg. web. cern. ch/LEPEWWG/

[27] N. B. Schmidt, S. A. Cetin, S. Istin and S. Sultansoy, arXiv:0908.2653 [hep-ph].

[28] A. Lister [CDF Collaboration], arXiv:0810.3349 [hepex]; T. Aaltonen et al. [The CDF Collaboration], arXiv: 0912.1057 [hep-ex].

[29] S. L. Glashow and S. Weinberg, Phys. Rev. D 15, 1958 (1977).

[30] For the analysis in the three generation model, see, e.g., S. Nie and M. Sher, Phys. Lett. B 449, 89 (1999) [arXiv: hep-ph/9811234]; S. Kanemura, T. Kasai and Y. Okada, Phys. Lett. B 471, 182 (1999) [arXiv:hep-ph/9903289].

[31] C. T. Hill, C. N. Leung and S. Rao, Nucl. Phys. B 262, 517 (1985).

[32] S. Kanemura, Y. Okada, E. Senaha and C. P. Yuan, Phys. Rev. D 70, 115002 (2004) [arXiv:hep-ph/0408364].

[33] See, e.g., K. Cheung and O. C. W. Kong, Phys. Rev. D 68, 053003 (2003); A. Wahab El Kaffas, P. Osland and O. M. Ogreid, Phys. Rev. D 76, 095001 (2007).

[34] M. Misiak et al., Phys. Rev. Lett. 98, 022002 (2007) [arXiv: hep-ph/0609232].

[35] O. Deschamps, S. Descotes-Genon, S. Monteil, V. Niess, S. T'Jampens and V. Tisserand, arXiv:0907.5135 [hep$\mathrm{ph}$.

[36] A. Denner, R. J. Guth, W. Hollik and J. H. Kuhn, Z. Phys. C 51, 695 (1991).

[37] H. E. Haber and H. E. Logan, Phys. Rev. D 62, 015011 (2000) [arXiv:hep-ph/9909335].

[38] R. Barate et al. [LEP Working Group for Higgs boson searches and ALEPH Collaboration], Phys. Lett. B 565, 61 (2003) [arXiv:hep-ex/0306033].

[39] H. Komatsu, Prog. Theor. Phys. 67, 1177 (1982).

[40] T. Cuhadar-Donszelmann, M. K. Unel, V. E. Ozcan, S. Sultansoy and G. Unel, JHEP 0810, 074 (2008) [arXiv:0806.4003 [hep-ph]]; V. E. Ozcan, S. Sultansoy and G. Unel, J. Phys. G 36, 095002 (2009) [arXiv: 0903.3177 [hep-ex]].

[41] S. Bar-Shalom, G. Eilam and A. Soni, arXiv:1001.0569 [hep-ph].

[42] V. D. Barger, J. L. Hewett and R. J. N. Phillips, Phys. Rev. D 41, 3421 (1990); Y. Grossman, Nucl. Phys. B 426, 355 (1994) [arXiv:hep-ph/9401311]; M. Aoki, S. Kanemura, K. Tsumura and K. Yagyu, Phys. Rev. D 80, 015017 (2009) [arXiv:0902.4665 [hep-ph]]; S. Su 
and B. Thomas, Phys. Rev. D 79, 095014 (2009) [arXiv: 0903.0667 [hep-ph]]; H. E. Logan and D. MacLennan, Phys. Rev. D 79, 115022 (2009) [arXiv:0903.2246 [hep$\mathrm{ph}]$.

[43] S. Bertolini and A. Sirlin, Phys. Lett. B 257, 179 (1991); E. Gates and J. Terning, Phys. Rev. Lett. 67, 1840 (1991); B. A. Kniehl and H. G. Kohrs, Phys. Rev. D 48, 225 (1993); see also the last two of Ref. [7].

[44] M. Bobrowski, A. Lenz, J. Riedl and J. Rohrwild, Phys. Rev. D 79, 113006 (2009) [arXiv:0902.4883 [hep-ph]].

[45] M. S. Chanowitz, Phys. Rev. D 79, 113008 (2009) [arXiv:0904.3570 [hep-ph]].

[46] S. Kanemura, S. Kiyoura, Y. Okada, E. Senaha and C. P. Yuan, Phys. Lett. B 558, 157 (2003) [arXiv: hep-ph/0211308].

[47] U. Baur, T. Plehn and D. L. Rainwater, Phys. Rev. Lett. 89, 151801 (2002); Phys. Rev. D 67, 033003 (2003); see also, F. Gianotti et al., Eur. Phys. J. C 39, 293 (2005).

[48] V. A. Ilyin, A. E. Pukhov, Y. Kurihara, Y. Shimizu and T. Kaneko, Phys. Rev. D 54, 6717 (1996) [arXiv: hep-ph/9506326]; G. Belanger et al., Phys. Lett. B 576, 152 (2003) [arXiv:hep-ph/0309010]; E. Asakawa,
D. Harada, S. Kanemura, Y. Okada and K. Tsumura, Phys. Lett. B 672, 354 (2009) [arXiv:0809.0094 [hep$\mathrm{ph}]$.

[49] W. S. Hou, M. Nagashima, G. Raz and A. Soddu, JHEP 0609, 012 (2006) [arXiv:hep-ph/0603097]; W. S. Hou, M. Nagashima and A. Soddu, Phys. Rev. Lett. 95, 141601 (2005) [arXiv:hep-ph/0503072]; W. S. Hou, H. n. Li, S. Mishima and M. Nagashima, Phys. Rev. Lett. 98, 131801 (2007) [arXiv:hep-ph/0611107]; W. S. Hou, M. Nagashima and A. Soddu, Phys. Rev. D 76, 016004 (2007) [arXiv:hep-ph/0610385].

[50] A. Soni, A. K. Alok, A. Giri, R. Mohanta and S. Nandi, arXiv:0807.1971 [hep-ph].

[51] M. S. Carena, A. Megevand, M. Quiros and C. E. M. Wagner, Nucl. Phys. B 716, 319 (2005) [arXiv:hep-ph/0410352]; Y. Kikukawa, M. Kohda and J. Yasuda, Prog. Theor. Phys. 122, 401 (2009) [arXiv:0901.1962 [hep-ph]].

[52] M. S. Chanowitz, Phys. Rev. Lett. 69, 2037 (1992); Phys. Lett. B 352, 376 (1995) [arXiv:hep-ph/9503458]. 